\title{
Nonlinear dynamics of micromechanical resonator arrays for mass sensing
}

\author{
S. Baguet $\bowtie$ · V-N. Nguyen · C. Grenat · C-H. Lamarque · R. Dufour
}

Received: date / Accepted: date

\begin{abstract}
This paper investigates the mass-sensing capability of an array of a few identical electrostatically actuated microbeams, as a first step toward the implementation of arrays of thousands of such resonant sensors. A reducedorder model is considered and Taylor series are used to simplify the nonlinear electrostatic force. Then the Harmonic Balance Method associated with the Asymptotic Numerical Method, as well as time-integration or averaging methods are applied to this model and their results are compared. In this paper, two- and three-beam arrays are studied. The predicted responses exhibit complex branches of solutions with additional loops due to the influence of adjacent beams. Moreover, depending on the applied voltages, the solutions with and without added mass exhibit large differences in amplitude which can be used for detection. For symmetric configurations, the symmetry breaking induced by an added mass is exploited to improve mass sensing.
\end{abstract}

Keywords MEMS · mass sensing - resonator array · nonlinear dynamics · bifurcation · symmetry breaking . electrostatic actuation

\section{Introduction}

Today, micro and nano beam resonators are widely used as sensors in several domains due to their high accuracy

S. Baguet $\bowtie$ (corresponding author)

V-N. Nguyen $\cdot$ C. Grenat $\cdot$ R. Dufour

Univ Lyon, INSA-Lyon, CNRS UMR5259, LaMCoS, F-69621, France

E-mail: Sebastien.Baguet@insa-lyon.fr

E-mail: Van-nghi.Nguyen@insa-lyon.fr

E-mail: Clement.Grenat@insa-lyon.fr

E-mail: Regis.Dufour@insa-lyon.fr

C-H. Lamarque

Univ Lyon, ENTPE, CNRS UMR5513, LTDS, F-695181, France

E-mail: Claude.Lamarque@entpe.fr combined with a reduced size, a target application being 5 the measure of infinitesimal mass in biological environment: biomolecule, DNA, protein, enzyme, etc. A lot of research works focus on improving the sensitivity of a single mass sensor by reducing the sensor size, increasing the signal-tonoise ratio or exciting the sensor in nonlinear regime $[1,2$, 10 3]. Some researchers considered higher bending modes of vibration [4,5] while other researchers showed that using the first torsional mode is more efficient than the first bending mode [6]. Eichler et al. [7] examined the role played by symmetry breaking due to the bending effect in a mechani15 cal resonator.

Exploitation of nonlinear phenomena to improve performance has recently received significant attention. A review of past, present and future research on the use of nonlinear phenomena in micro-electro-mechanical systems (MEMS) 20 was proposed by Rhoads et al. [8]. Younis et al. [9] used the pull-in instability phenomenon of a microresonator to design a new method for mass detection in which the jump to pullin acts as a switch with a mass threshold. Ruzziconi et al. [10] studied a microbeam under axial load and electric exci25 tation. The robustness of stable motions, the disappearance of main attractors and potential jumps to pull-in under disturbances were studied with dynamical integrity concepts. Kumar et al. [11] provided a mass sensing method relying on jumps at limit points of the nonlinear frequency response. 30 Nguyen et al. [12] improved the method with an automatic reinitialization exploiting hysteresis cyles and paving the way for real-time detection. Zhang et al. [13] and Thomas et al. [14] studied the parametric resonance and showed that the sensitivity is highly increased due to the sharpness of am35 plitude transition in this regime. Other research focused on canceling the nonlinearities. In [15], Kacem et al. showed that the combination of simultaneous resonance and compensation of nonlinear terms can lead to dynamic stabiliza- 
tion of the nanoresonator even for amplitudes of vibration ${ }_{40}$ close to the gap.

At present time, using MEMS resonators makes the detection of very tiny mass possible. For example, a mass sensor operating at ultra-high frequency $(1.3 \mathrm{GHz})$ is capable of detecting $10^{-18} \mathrm{~g}$ [16] and recently a MEMS-based mass ${ }_{45}$ spectrometer accessed masses above $500 \mathrm{k} D a(1 D a=1.66 \times$ $10^{-27} \mathrm{~kg}$ ) [17]. However, it is difficult to detect simultaneously many particles and separate one of them in order to drop it onto a resonant microbeam. Detecting several masses at the same time legitimates the research on resonator arrays.

50 Current technology enables the fabrication of large arrays composed of a few as well as of thousands of MEMS, the dynamics of which results from electrical, magnetical and mechanical couplings. The dynamical behavior of such arrays is studied experimentally or theoretically in several 55 references $[18,19,20]$. Theoretical models are still not fully established and exploited because of the complexity of nonlinear couplings and variety of phenomena. Some recent works started dealing with these problems. For example, Porfiri [21] showed that the vibrational properties of an array of ${ }_{60}$ identical microplates can be tuned by properly adjusting the bias voltage applied across adjacent microplates. Karabalin et al. [22] considered an array of two nanoresonators coupled by an elastic force and independently excited around their resonant frequencies. They showed that the linear and ${ }_{65}$ weakly nonlinear responses of a nano-resonator can be modified by the excitation of the other nano-resonators. When two resonators are strongly excited in their nonlinear domain, the response curves become more complex. In the same way, Lifshitz et al [19] showed that increasing the 70 number of oscillators makes the branches of solutions more and more numerous.

In addition to the numerous branches of solutions, modal couplings also occur in the dynamics of a beam array. For example Gutschmidt et al. studied a beam array in a 1:1 res-

75 onance configuration as well as 3:1 internal resonances near pull-in [23, 24,25,26]. Kambali et al [27] focused on understanding the couplings of several modal frequencies and their tuning mechanisms. In the study, modal frequencies were tuned by adjusting the continuous actuation voltage in 80 order to rescale the beam array into a specific configuration. Studies on modal couplings and internal resonances not only permit a better understanding of the dynamics of beam arrays, but also give some insights on specific configurations such as the symmetric one.

${ }_{85}$ In this paper, an array of $n$ identical clamped-clamped beams in bending is considered in order to study the response change due to a very small added mass. This response change is analyzed numerically for various numbers of beams and several configuration of the beam array such as

90 asymmetric and symmetric ones. The $n$ beams of the array are coupled only by electrostatic forces and exhibit complex

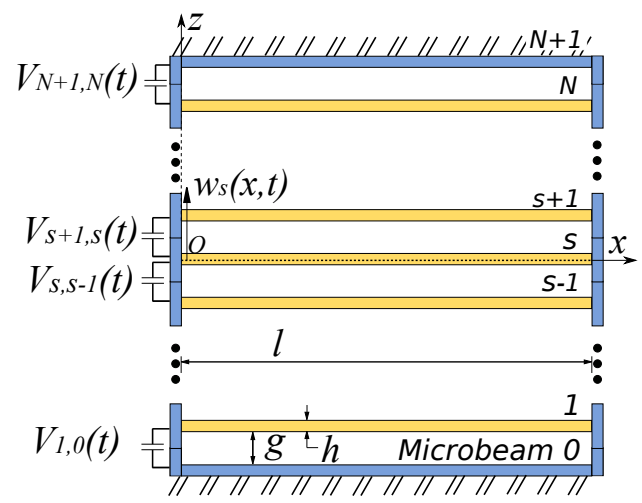

Fig. 1 Model of the clamped-clamped microbeam array.

dynamical behaviors which are used for mass sensing. This paper is organized as follows. In Section 2, a reduced-order model for an array of $n$ clamped-clamped beams is consid${ }_{95}$ ered. It is obtained through Galerkin expansion onto a finite number of linear eigenmodes and solved numerically by means of the Harmonic Balance Method (HBM) combined with the Asymptotic Numerical Method (ANM). In Section 3 , the case of a two-beam array is investigated. The response 100 change due to the influence of the adjacent beam is analyzed. Approximated solutions are discussed and mass detection mechanisms are introduced according to the specific dynamical behaviors of the beams. In Section 4, a three-beam array is examined. Two cases are distinguished: asymmetric 105 and symmetric actuations. An original mass sensing principle based on the symmetry breaking event in a symmetrical configuration of the beam array is presented. An analysis related to location, possible detection as well as quantification of the added mass is conducted. Conclusions are drawn in ${ }_{110}$ Section 5.

\section{Model}

Let an array of $n$ clamped-clamped beams be considered as sketched in Fig. 1. All the beams are assumed to have identical dimensions ( length $l$, width $b$, height $h$ and moment of 115 inertia $I$ ) and identical material properties (Young's modulus $E$ and material density $\rho$ ). Each beam is subject to the electrostatic forces due to its adjacent beams. The two beams at both ends of the array (beams \#0 and \#(n+1)) are prevented from any lateral motion and just serve for actuation. Let $g$ ${ }_{120}$ be the gap between two adjacent beams.

\subsection{Case without added mass}

Let the lateral deflection be in the $x z$ plane and let $\tilde{w}_{s}$ be the lateral displacement along the $z$-axis of the $\mathrm{s}^{\text {th }}$ beam and $V_{s, s+1}=V_{d c_{s, s+1}}+V_{a c_{s, s+1}} \cos (\tilde{\Omega} \tilde{t})$ the voltage between ${ }_{22} s^{\text {th }}$ and $(s+1)^{\text {th }}$ beams. $\tilde{\Omega}, \tilde{t}$ denote physical frequency and 
time respectively. $V_{d c}, V_{a c}$ denote bias and alternative volt- ${ }_{145}$ Let

ages respectively. The $\mathrm{s}^{\text {th }}$ beam is subject to two electrostatic forces

$$
\begin{aligned}
& X_{s}(x, t)=w_{s+1}(x, t)-w_{s}(x, t), \\
& b_{k}^{s}(t)=a_{k}^{s+1}(t)-a_{k}^{s}(t),
\end{aligned}
$$

- the first one due to the $(s+1)^{\text {th }}$ beam

$$
f_{s, s+1}^{e}=\frac{1}{2} b \varepsilon_{0} C_{n} \frac{V_{s, s+1}^{2}}{\left(g+\tilde{w}_{s+1}-\tilde{w}_{s}\right)^{2}} .
$$

130 - the second one due to the $(s-1)^{\text {th }}$ beam

$$
f_{s-1, s}^{e}=-\frac{1}{2} b \varepsilon_{0} C_{n} \frac{V_{s-1, s}^{2}}{\left(g+\tilde{w}_{s}-\tilde{w}_{s-1}\right)^{2}} .
$$

$\varepsilon_{0}, C_{n}$ are the dielectric constant and fringing field coefficient respectively. Consequently the equation of motion of the $s^{\text {th }}$ beam in a $n$-beam array is:

$$
\begin{aligned}
& E I \frac{\partial^{4} \tilde{w}_{s}(\tilde{x}, \tilde{t})}{\partial \tilde{x}^{4}}+\rho b h \frac{\partial^{2} \tilde{w}_{s}(\tilde{x}, \tilde{t})}{\partial \tilde{t}^{2}}+\tilde{c} \frac{\partial \tilde{w}_{s}(\tilde{x}, \tilde{t})}{\partial \tilde{t}}- \\
& {\left[\tilde{N}_{s}+\frac{E b h}{2 l} \int_{0}^{l}\left(\frac{\partial \tilde{w}_{s}(\tilde{x}, \tilde{t})}{\partial \tilde{x}}\right)^{2} \mathrm{~d} \tilde{x}\right] \frac{\partial^{2} \tilde{w}_{s}(\tilde{x}, \tilde{t})}{\partial \tilde{x}^{2}}} \\
& \quad=\frac{1}{2} \varepsilon_{0} \frac{b C_{n} V_{s, s+1}^{2}}{\left(g+\tilde{w}_{s+1}-\tilde{w}_{s}\right)^{2}}-\frac{1}{2} \varepsilon_{0} \frac{b C_{n} V_{s-1, s}^{2}}{\left(g+\tilde{w}_{s}-\tilde{w}_{s-1}\right)^{2}}
\end{aligned}
$$

with $s=1, . ., n$ and $\tilde{N}_{s}$ the residual axial force acting on the $135 s^{\text {th }}$ beam and resulting from an externally applied load or from manufacturing stress. Beams \#0 and \# $(n+1)$ are totally clamped, so:

$\tilde{w}_{0}(\tilde{x}, \tilde{t})=\tilde{w}_{n+1}(\tilde{x}, \tilde{t})=0$.

Introducing the following non-dimensional parameters

$w_{s}=\frac{\tilde{w}_{s}}{g} ; \quad x=\frac{\tilde{x}}{l} ; \quad t=\frac{\tilde{t}}{\tau} ; \quad \Omega=\tilde{\Omega} \tau ;$

be the relative displacement and generalized coordinates between two consecutive beams. The relations between $X_{s}(x, t)$ and $b_{k}^{s}(x, t)$ are:

$1+w_{s+1}-w_{s}=1+\sum_{k=1}^{N_{m}} \phi_{k}(x) b_{k}^{s}(t)=1+X_{s}$,

$1+w_{s}-w_{s-1}=1+\sum_{k=1}^{N_{m}} \phi_{k}(x) b_{k}^{s-1}(t)=1+X_{s-1}$.

Multiplying by the denominator or using Taylor series are 150 two methods to treat the electrostatic force in Eq. (5). Using the multiplication by $\left(1+w_{s+1}-w_{s}\right)^{2} \times\left(1+w_{s}-w_{s-1}\right)^{2}$ in Eq. (5) introduces additional terms with strong couplings and increases the complexity of the problem. On the other hand, when using truncated Taylor series, the accuracy is 55 usually bad at high amplitude [28]. Since the resonators will operate at small amplitudes only $\left(\max w_{s} \leq 0.3\right)$, a seventhorder Taylor series is used [29]:

$$
\begin{aligned}
\frac{1}{\left(1+w_{s+1}-w_{s}\right)^{2}} \simeq & 1-2 X_{s}+3 X_{s}^{2} \\
& -4 X_{s}^{3}+5 X_{s}^{4}-6 X_{s}^{5}+7 X_{s}^{6}-8 X_{s}^{7}, \\
\frac{1}{\left(1+w_{s}-w_{s-1}\right)^{2}} \simeq & 1-2 X_{s-1}+3 X_{s-1}^{2}-4 X_{s-1}^{3} \\
& +5 X_{s-1}^{4}-6 X_{s-1}^{5}+7 X_{s-1}^{6}-8 X_{s-1}^{7}
\end{aligned}
$$

$c=\frac{\tilde{c} l^{4}}{E I \tau} ; \quad N_{s}=\frac{\tilde{N}_{s} l^{2}}{E I} ; \quad \alpha_{1}=6\left(\frac{g}{h}\right)^{2} ; \quad \alpha_{2}=6 C_{n} \frac{\varepsilon_{0} l^{4}}{E h^{3} g^{3}}$,

in Eq. (3), yields:

$$
\begin{array}{r}
\frac{\partial^{4} w_{s}}{\partial x^{4}}+\frac{\partial^{2} w_{s}}{\partial t^{2}}+c \frac{\partial w_{s}}{\partial t}-\left[N_{s}+\alpha_{1} \int_{0}^{1}\left(\frac{\partial w}{\partial x}\right)^{2} \mathrm{~d} x\right] \frac{\partial^{2} w_{s}}{\partial x^{2}} \\
=\alpha_{2} \frac{V_{s, s+1}^{2}}{\left(1+w_{s+1}-w_{s}\right)^{2}}-\alpha_{2} \frac{V_{s-1, s}^{2}}{\left(1+w_{s}-w_{s-1}\right)^{2}}
\end{array}
$$

${ }_{140}$ with $s=1, . ., n$ and the boundary conditions being:

$$
\begin{aligned}
& w_{s}(0, t)=w_{s}(1, t)=\frac{\partial w_{s}}{\partial x}(0, t)=\frac{\partial w_{s}}{\partial x}(1, t)=0, \\
& w_{0}(x, t)=w_{n+1}(x, t)=0
\end{aligned}
$$

By assumption, the $n$ identical beams have identical linear undamped eigenmodes $\phi_{k}$. A Galerkin expansion of the lateral displacement $w_{s}$ is expressed with the first $N_{m}$ modes:

$$
w_{s}(x, t) \approx \sum_{k=1}^{N_{m}} \phi_{k}(x) a_{k}^{s}(t)
$$

The Galerkin procedure requires to multiply Eq. (5) by $\phi_{i}$ $\left(i=1, . ., N_{m}\right)$ and to integrate the result from 0 to 1 to ob160 tain the second-order differential equations in time which are written in matrix form as follows:

$$
\begin{aligned}
\ddot{\boldsymbol{a}}^{s}+\boldsymbol{C}_{0} \dot{\boldsymbol{a}}^{s}+\boldsymbol{K}_{0} \boldsymbol{a}^{s}-\left(N+\alpha_{1} T_{2}^{s}\left(\boldsymbol{a}^{s}\right)\right) \boldsymbol{K}_{T} \boldsymbol{a}^{s}= \\
\alpha_{2} V_{s, s+1}^{2} \boldsymbol{Q}_{0}+\alpha_{2} V_{s, s+1}^{2}\left[\boldsymbol{Q}_{1}+\boldsymbol{Q}_{2}^{s}\left(\boldsymbol{b}^{s}\right)+\boldsymbol{Q}_{3}^{s}\left(\boldsymbol{b}^{s}\right)+\boldsymbol{Q}_{4}^{s}\left(\boldsymbol{b}^{s}\right)\right. \\
\left.+\boldsymbol{Q}_{5}^{s}\left(\boldsymbol{b}^{s}\right)+\boldsymbol{Q}_{6}^{s}\left(\boldsymbol{b}^{s}\right)+\boldsymbol{Q}_{7}^{s}\left(\boldsymbol{b}^{s}\right)\right] \boldsymbol{b}^{s}-\alpha_{2} V_{s-1, s} V_{s-1, s}^{2} \boldsymbol{P}_{0} \\
-\alpha_{2} V_{s-1, s}^{2}\left[\boldsymbol{P}_{1}+\boldsymbol{P}_{2}^{s}\left(\boldsymbol{b}^{s-1}\right)+\boldsymbol{P}_{3}^{s}\left(\boldsymbol{b}^{s-1}\right)+\boldsymbol{P}_{4}^{s}\left(\boldsymbol{b}^{s-1}\right)\right. \\
\left.+\boldsymbol{P}_{5}^{s}\left(\boldsymbol{b}^{s-1}\right)+\boldsymbol{P}_{6}^{s}\left(\boldsymbol{b}^{s-1}\right)+\boldsymbol{P}_{7}^{s}\left(\boldsymbol{b}^{s-1}\right) \boldsymbol{b}^{s-1}\right]
\end{aligned}
$$

where $s=1, . ., n, \boldsymbol{a}^{s}=\left[a_{1}^{s}, a_{2}^{s}, \ldots, a_{N_{m}}^{s}\right]$ and $\boldsymbol{a}^{n+1}=\boldsymbol{a}^{0}=0$ resulting from Eq. (7). The matrices in Eq. (15) are detailed in Appendix A.

165 The HBM associated with the ANM (HBM+ANM) [30] is used to solve Eq. (15). The ANM is preferred to a more 
conventional Newton-Raphson method because of the robustness of the ANM-based continuation and its capability to follow very complicated solution branches. First, Eq. (15) 170 must be recast in quadratic form by introducing the following set of auxiliary variables:

$\begin{array}{ll}\boldsymbol{y}^{s}=\dot{\boldsymbol{a}}^{s} & \text { size } \\ \boldsymbol{z}^{s}=\dot{\boldsymbol{y}}^{s} & N_{m} \\ \boldsymbol{n} \boldsymbol{u}^{s}=\boldsymbol{b}^{s}=\boldsymbol{a}^{s+1}-\boldsymbol{a}^{s} & N_{m} \\ \boldsymbol{b} \boldsymbol{u}^{s}=\boldsymbol{b}^{s-1}=\boldsymbol{a}^{s}-\boldsymbol{a}^{s-1} & N_{m} \\ \boldsymbol{n u} 2^{s}=\boldsymbol{n u} \boldsymbol{u}^{s} \times \boldsymbol{n u} \boldsymbol{u}^{s} & N_{m} \\ \boldsymbol{n u} 3^{s}=\boldsymbol{n u} 2^{s} \times \boldsymbol{n} \boldsymbol{u}^{s} & N_{m}^{2} \\ \boldsymbol{b} \boldsymbol{u} 2^{s}=\boldsymbol{b} \boldsymbol{u}^{s} \times \boldsymbol{b} \boldsymbol{u}^{s} & N_{m}^{3} \\ \boldsymbol{b u} 3^{s}=\boldsymbol{b u} 2^{s} \times \boldsymbol{b} \boldsymbol{u}^{s} & N_{m}^{2} \\ \boldsymbol{A} \boldsymbol{Q}^{s}=\boldsymbol{Q}_{1}+\boldsymbol{Q}_{2}^{s}+\boldsymbol{Q}_{3}^{s}+\boldsymbol{Q}_{4}^{s}+\boldsymbol{Q}_{5}^{s}+\boldsymbol{Q}_{6}^{s}+\boldsymbol{Q}_{7}^{s} & N_{m}^{2} \\ \boldsymbol{B} \boldsymbol{P}^{s}=\boldsymbol{P}_{1}+\boldsymbol{P}_{2}^{s}+\boldsymbol{P}_{3}^{s}+\boldsymbol{P}_{4}^{s}+\boldsymbol{P}_{5}^{s}+\boldsymbol{P}_{6}^{s}+\boldsymbol{P}_{7}^{s} & N_{m}^{2} \\ v 1^{s}=\alpha_{2} V_{s, s+1}^{2} & 1 \\ v 2^{s}=\alpha_{2} V_{s-1, s}^{2} & 1 \\ t 2^{s}=T_{2}^{s}\left(a^{s}\right) & 1 \\ \boldsymbol{A} \boldsymbol{A} \boldsymbol{Q}^{s}=v 1^{s} \boldsymbol{A} \boldsymbol{Q}^{s} & N_{m}^{2} \\ \boldsymbol{B} \boldsymbol{B} \boldsymbol{P}^{s}=v 2^{s} \boldsymbol{B} \boldsymbol{P}^{s} & N_{m}^{2} \\ & \end{array}$

so the new form of Eq. (15) adapted to HBM+ANM is:

$$
\begin{aligned}
& \dot{\boldsymbol{a}}^{s}=\quad \boldsymbol{y}^{s} \\
& \dot{\boldsymbol{y}}^{s}=\quad \boldsymbol{z}^{s} \\
& 0=\quad \boldsymbol{n u} \boldsymbol{u}^{s}- \\
& \left(a^{s+1}-a^{s}\right) \\
& \begin{array}{c}
\boldsymbol{b u}- \\
\left(\boldsymbol{a}^{s}-\boldsymbol{a}^{s-1}\right)
\end{array} \\
& 0=\quad \boldsymbol{n u} 2^{s}-\quad \boldsymbol{n u} \boldsymbol{u}^{s} \times \boldsymbol{n u} \boldsymbol{u}^{s} \\
& 0=\quad \mathbf{n u} 3^{s}-\quad \boldsymbol{n u} 2^{s} \times \boldsymbol{n u} \boldsymbol{u}^{s} \\
& 0=\quad \boldsymbol{b u} 2^{s}-\quad \boldsymbol{b u} \boldsymbol{u}^{s} \times \boldsymbol{b} \boldsymbol{u}^{s} \\
& 0=\quad \boldsymbol{b u} 3^{s}-\quad \boldsymbol{b u} 2^{s} \times \boldsymbol{b u} \boldsymbol{u}^{s} \\
& 0=-Q_{1} \quad+A Q^{s}-Q_{2}^{s} \quad-Q_{5}^{s}-Q_{6}^{s}
\end{aligned}
$$

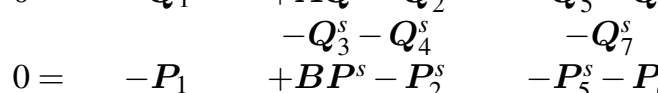

$$
\begin{aligned}
& \begin{array}{ll}
-\boldsymbol{P}_{3}^{s}-\boldsymbol{P}_{4}^{s} & -\boldsymbol{P}_{7}^{s}
\end{array} \\
& 0=\alpha_{2} V_{s, s+1}^{2} \quad-v 1^{s} \\
& 0=\alpha_{2} V_{s-1, s}^{2} \quad-v 2^{s} \\
& 0=\quad t 2^{s} \\
& 0=\quad A A Q \\
& 0=\quad B B P \\
& 0=\quad \boldsymbol{z}^{s}+\boldsymbol{C}_{0} \boldsymbol{y}^{s}+\boldsymbol{K}_{0} \boldsymbol{a}^{s}-\boldsymbol{A} \boldsymbol{A} \boldsymbol{Q} \times \boldsymbol{n u} \\
& -v 1^{s} Q_{0}+\quad+B B P \times b u \\
& \underbrace{}_{m\left(\dot{\boldsymbol{X}}^{s}\right)} \underbrace{}_{l_{0}(\Omega)} \underbrace{v 2^{s} \boldsymbol{P}_{0}-N \boldsymbol{K}_{T} \boldsymbol{a}^{s}}_{+l\left(\boldsymbol{X}^{s}\right)} \underbrace{-\alpha_{1} t 2^{s} \boldsymbol{K}_{T} \boldsymbol{a}^{s}}_{+q\left(\boldsymbol{X}^{s}, \boldsymbol{X}^{s}\right)}
\end{aligned}
$$

where: $\boldsymbol{X}^{s}=\left(\boldsymbol{a}^{s}, \boldsymbol{y}^{s}, \boldsymbol{z}^{s}, \boldsymbol{n u ^ { s }}, \boldsymbol{b u} \boldsymbol{u}^{s}, \boldsymbol{n u} 2^{s}, \boldsymbol{n u} 3^{s}, \boldsymbol{b u} 2^{s}, \boldsymbol{b u} 3^{s}\right.$, $\left.\boldsymbol{A} \boldsymbol{Q}^{s}, \boldsymbol{B} \boldsymbol{P}^{s}, \boldsymbol{A} \boldsymbol{A} \boldsymbol{Q}^{s}, \boldsymbol{B} \boldsymbol{B} \boldsymbol{P}^{s}, v 1^{s}, v 2^{s}, t 2^{s}\right)^{T}$ is the unknown 175 vector of size $5 N_{m}+3+6 N_{m}^{s}+2 N_{m}^{3}$. For the array of $n$ beams, with $s=1, . ., n, \boldsymbol{X}=\left(\boldsymbol{X}^{1}, \boldsymbol{X}^{2}, \boldsymbol{X}^{3}, . ., \boldsymbol{X}^{n}, \Omega\right)^{T}$ is the unknown vector of size $n \times\left(5 N_{m}+3+6 N_{m}^{s}+2 N_{m}^{3}\right)+1$.

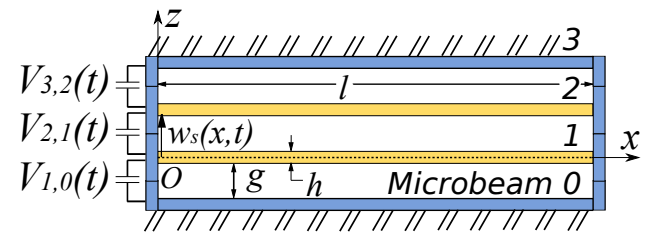

Fig. 2 Array of 2 clamped-clamped microbeams.

Table 1 Actuation voltages in the two-beam array

\begin{tabular}{|c|c|c|c|}
\hline Design & $V_{d c_{10}}(V)$ & $V_{d c_{21}}(V)$ & $V_{d c_{32}}(V)$ \\
& $V_{a c_{10}}(V)$ & $V_{a c_{21}}(V)$ & $V_{a c_{32}}(V)$ \\
\hline$\# 1$ & 0.3 & 0.45 & 0.6 \\
& 0.3 & 0.45 & 0.6 \\
\hline
\end{tabular}

\subsection{Case with added mass}

The presence of a small mass $m_{p}$ at position $x_{0}$ on the $s_{0}{ }^{\text {th }}$ 180 beam introduces the extra term $\mu_{0} \ddot{\boldsymbol{a}}^{s}$ in Eq. (15), the matrix $\mu_{0}$ being defined as follows:

$$
\mu_{0 i j}=\delta_{s_{0}}(s) m \phi_{i}\left(x_{0}\right) \phi_{j}\left(x_{0}\right), \quad i, j=1, . ., N_{m}
$$

with $\delta_{s_{0}}(s)=1$ when $s=s_{0}$ and $\delta_{s_{0}}(s)=0$ otherwise.

The mass ratio between the added mass $m_{p}$ and the mass of the beam is:

$m=\frac{m_{p}}{\rho b h l}$

${ }_{185}$ It is used to replace the physical value of the added mass by a dimensionless value in the following sections.

\section{Two-beam array}

A two-beam array is considered, see Fig. (2). All beams are identical with the following material and design parameters: $190 h=300 \mathrm{~nm}, b=160 \mathrm{~nm}, l=10 \mu \mathrm{m}, E=1.69 \times 10^{11} \mathrm{~N} / \mathrm{m}^{2}$, $\rho=2330 \mathrm{~kg} / \mathrm{m}^{3}$, the quality factor $Q=5000$ and identical gaps $g=200 \mathrm{~nm}$ between two adjacent beams. The voltages in Table (1) are considered. It is worth mentioning here that considering the electrostatic coupling between two consec195 utive beams only is a valid assumption. Indeed, the distance between beams \#0 and \#2 is equal to two times the gap $g$ plus the beam width $b$, i.e., $3.5 g$ since $b=1.5 g$ for the considered design. As a result, the electrostatic forces beyond the gap $g$ are actually 12.25 times lower, i.e., one order lower 200 than the force between two consecutive beams.

First, it is necessary to assess the convergence of the beam responses with different Taylor-series orders $T$ and several modes.

Fig. 3 shows the nonlinear forced response curves obtained with different Taylor series orders and several vibration modes by using HBM+ANM. For all the figures involving curves in colors, the reader is referred to the online version of this paper. First, computations using third $(T=3)$, 
fifth $(T=5)$ and seventh $(T=7)$ order Taylor series are 210 carried out with $N_{m}=1$ mode. The deviation between responses for $T=5$ and $T=7$ is negligible because the corresponding Taylor series are accurate at small amplitude. Then, the influence of the number of modes in the Galerkin expansion is considered by using a Taylor series at order 2157 with $N_{m}=1, N_{m}=2$ and $N_{m}=3$. As shown in Fig. 3, the response is converged with $N_{m}=2$ modes. Hence, using Taylor series at order 7 and $N_{m}=2$ modes provides good accuracy. However, the computation with several modes is very time consuming and complicated. For the series at or${ }_{220} \operatorname{der} 7$, the relative error in frequency between $N_{m}=1$ and $N_{m}=2$ modes is less than $5.10^{-6}$ in frequency and quantitatively the response behavior does not change. For each case, the additional loops $D_{1}-E_{1}$ (first beam) and $B_{2}-C_{2}$ (second beam) on the response curves are the same. Conse225 quently, Taylor series at order 7 and $N_{m}=1$ mode are used to simplify the numerical calculation without loss of accuracy.

In Fig. 3, responses are more complicated than in the case of a single beam and present additional loops. A quasi230 analytic solution obtained by the averaging method provides some explanations about the origin of these additional branches.

\subsection{Averaging method}

The beam lateral deflection is expanded on its fundamental mode only:

$$
\begin{aligned}
& w_{1}(x, t)=\phi_{1}(x) a_{11}(t) \\
& w_{2}(x, t)=\phi_{1}(x) a_{21}(t)
\end{aligned}
$$

${ }_{235}$ First-order Taylor series are used to simplify the analytic calculation:

$$
\begin{aligned}
& \frac{1}{\left(1+w_{s+1}-w_{s}\right)^{2}} \simeq 1-2\left(w_{s+1}-w_{s}\right), \\
& \frac{1}{\left(1+w_{s}-w_{s-1}\right)^{2}} \simeq 1-2\left(w_{s}-w_{s-1}\right) .
\end{aligned}
$$

Using the averaging method and considering the solutions $a_{11}(t), a_{21}(t)$ in following forms

$$
\begin{aligned}
& a_{11}=A_{11}(t) \cos (\Omega t)+B_{11}(t) \sin (\Omega t), \\
& a_{21}=A_{21}(t) \cos (\Omega t)+B_{21}(t) \sin (\Omega t),
\end{aligned}
$$

yields

$$
\begin{aligned}
\Omega \dot{A}_{11} & =-B_{11} \omega_{1} \varepsilon \sigma_{1}-\frac{1}{2} c A_{11} \Omega+\frac{3}{8} \beta_{11} A_{11}^{2} B_{11} \\
& +\frac{3}{8} \beta_{11} B_{11}^{3}+\frac{1}{8} \beta_{13} B_{11}+\left(\frac{1}{2} \delta_{11}+\frac{1}{8} \delta_{13}\right) B_{21}
\end{aligned}
$$

$$
\begin{aligned}
\Omega \dot{B}_{11} & =\frac{1}{2} c B_{11} \Omega+\frac{3}{8} \beta_{11} A_{11}^{3}+\frac{3}{8} \beta_{13} A_{11}+\frac{1}{2} \gamma_{12} \\
& +\frac{3}{8} \beta_{11} A_{11} B_{11}^{2}-A_{11} \omega_{1} \varepsilon \sigma_{1}+\left(\frac{1}{2} \delta_{11}+\frac{3}{8} \delta_{13}\right) A_{21}
\end{aligned}
$$
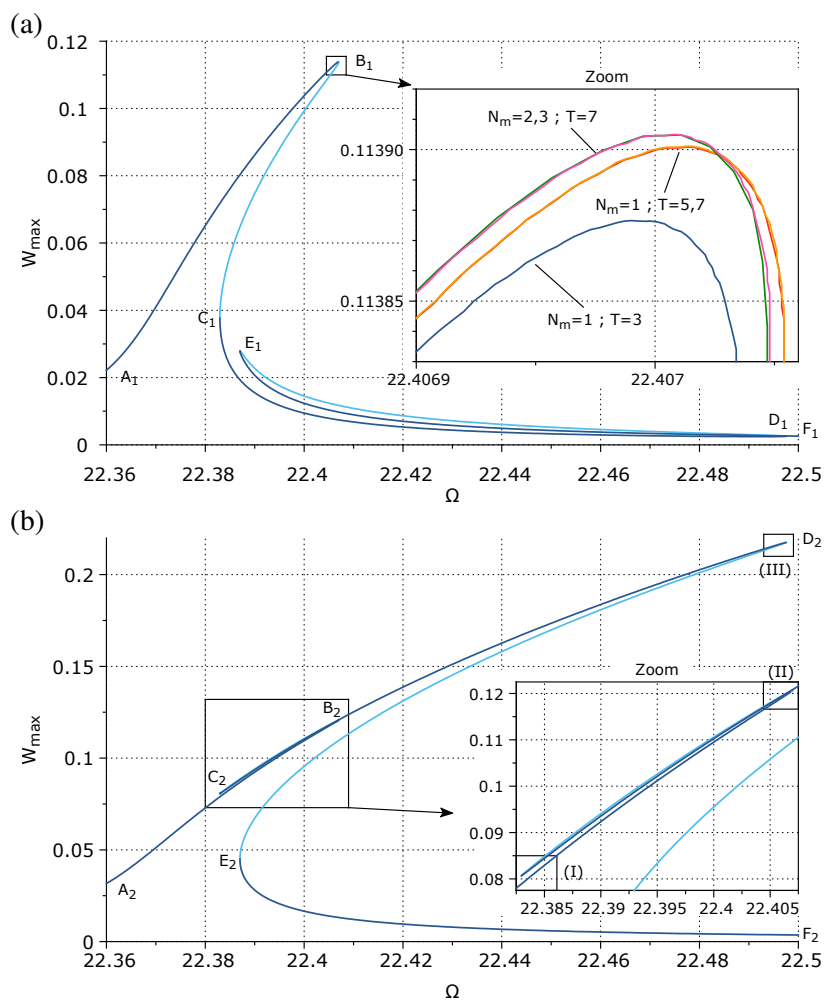

(c)

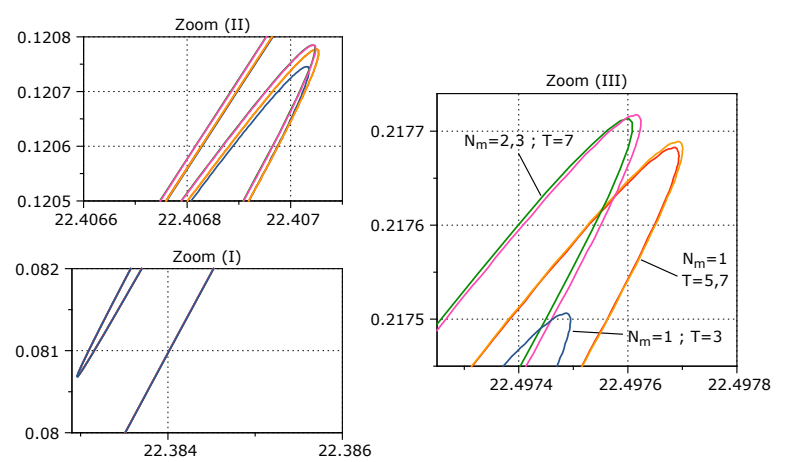

Fig. 3 Nonlinear forced response curves of a two-beam array without added mass by HBM+ANM method. Responses with third (blue), fifth (red) and seventh (orange) order Taylor series and $N_{m}=1$ mode; responses with seventh-order Taylor series with $N_{m}=2$ (pink) or $N_{m}=3$ (green) modes. Dark and light blue colors indicate stable and unstable solutions respectively. (a): Beam \#1, (b): Beam \#2, (c): zoom of (I), (II), (III).

$$
\begin{aligned}
\Omega \dot{A}_{21} & =-B_{21} \omega_{2} \varepsilon \sigma_{2}-\frac{1}{2} c A_{21} \Omega+\frac{3}{8} \beta_{21} A_{21}^{2} B_{21} \\
& +\frac{3}{8} \beta_{21} B_{21}^{3}+\frac{1}{8} \beta_{23} B_{21}+\left(\frac{1}{2} \delta_{21}+\frac{1}{8} \delta_{23}\right) B_{11} \\
\Omega \dot{B}_{21} & =\frac{1}{2} c B_{21} \Omega+\frac{3}{8} \beta_{21} A_{21}^{3}+\frac{3}{8} \beta_{23} A_{21}+\frac{1}{2} \gamma_{22} \\
& +\frac{3}{8} \beta_{21} A_{21} B_{21}^{2}-A_{21} \omega_{2} \varepsilon \sigma_{2}+\left(\frac{1}{2} \delta_{21}+\frac{3}{8} \delta_{23}\right) A_{11}
\end{aligned}
$$

The coefficients of Eqs. (24-27) are defined in Appendix B. In Eqs. (24-27), the coupling terms $\left(\frac{1}{2} \delta_{11}+\frac{1}{8} \delta_{13}\right) B_{21}$ 
245 and $\left(\frac{1}{2} \delta_{11}+\frac{1}{8} \delta_{13}\right) A_{21}$ represent the influence of the second beam on the first beam and the terms $\left(\frac{1}{2} \delta_{21}+\frac{1}{8} \delta_{23}\right) B_{11}$, $\left(\frac{1}{2} \delta_{21}+\frac{3}{8} \delta_{23}\right) A_{11}$ represent the influence of the first beam on the second beam. The steady-state motion occurs when $\dot{A}_{11}=\dot{B}_{11}=\dot{A}_{21}=\dot{B}_{21}=0$. This corresponds to an alge250 braic system of four nonlinear equations that can be solved numerically. The corresponding response curves are plotted in Fig. 4 and are in very good agreement with those of Fig. 3 obtained numerically by HBM+ANM.

\subsection{Response curve analysis}

255 Let Eqs. (24)-(27) be considered without these coupling terms. Therefore Eqs. (24)-(25) and (26)-(27) form two independent systems of equations. The responses plotted in Fig. 4 are similar to those of a single beam because no loops are present.

260 With coupling terms Eqs. (24)-(27) are dependent; therefore, they share the same bifurcation points and stability. Thus, the whole set of limits points on the two response curves without coupling terms are present on each response with coupling terms. Figure 4 shows that, in the presence 265 of coupling terms, points $B_{1}$ and $C_{1}$ on the first-beam response generate the loop $B_{2}-C_{2}$ on the second-beam response. Identically, points $D_{2}$ and $E_{2}$ on the second-beam response produce the loop $D_{1}-E_{1}$ on the first-beam response. The synchronization of stability leads to the appearance of 270 additional loops on the response curve.

Moreover, the first-beam amplitude changes from $W_{\max }=$ 0.15 to $W_{\max }=0.11$, whereas the second-beam amplitude is weakly affected. This is because the amplitude of the $B_{2}-$ $C_{2}$ generated loop is larger than the $D_{1}-E_{1}$ one. So, the 275 amount of energy transferred from the first beam to the second beam due to the coupling is larger than the amount of energy transferred from the second-beam to the first-beam.

\subsection{Comparing the results obtained by $\mathrm{HBM}+\mathrm{ANM}$ and time-integration methods.}

280 The results obtained by these two methods for the two-beam array without added mass are represented in Fig. 5 and fit well. However, because of the response complexity, it is impossible to obtain all the stable branches by time integration with a simple frequency sweep-up and sweep-down. ${ }_{285}$ For example, in Fig. 5, the stable branches $j E_{1}-j B_{1}$ (first beam) and $j E_{2}-B_{2}$ (second beam) are not obtained. For the first beam, with a frequency sweep-down the response path is $F_{1}-E_{1}-j E_{1}-C_{1}-j C_{1}-A_{1}$ where the letter $j$ indicates an amplitude jump. Unlike the single-beam response, 290 there are two jumps from $E_{1}$ to $j E_{1}$ and from $C_{1}$ to $j C_{1}$. For the second beam, the response path is $F_{2}-E_{2}-j E_{2}-A_{2}$ with a jump from $E_{2}$ to $j E_{2}$ and point $j E_{2}$ is not on the
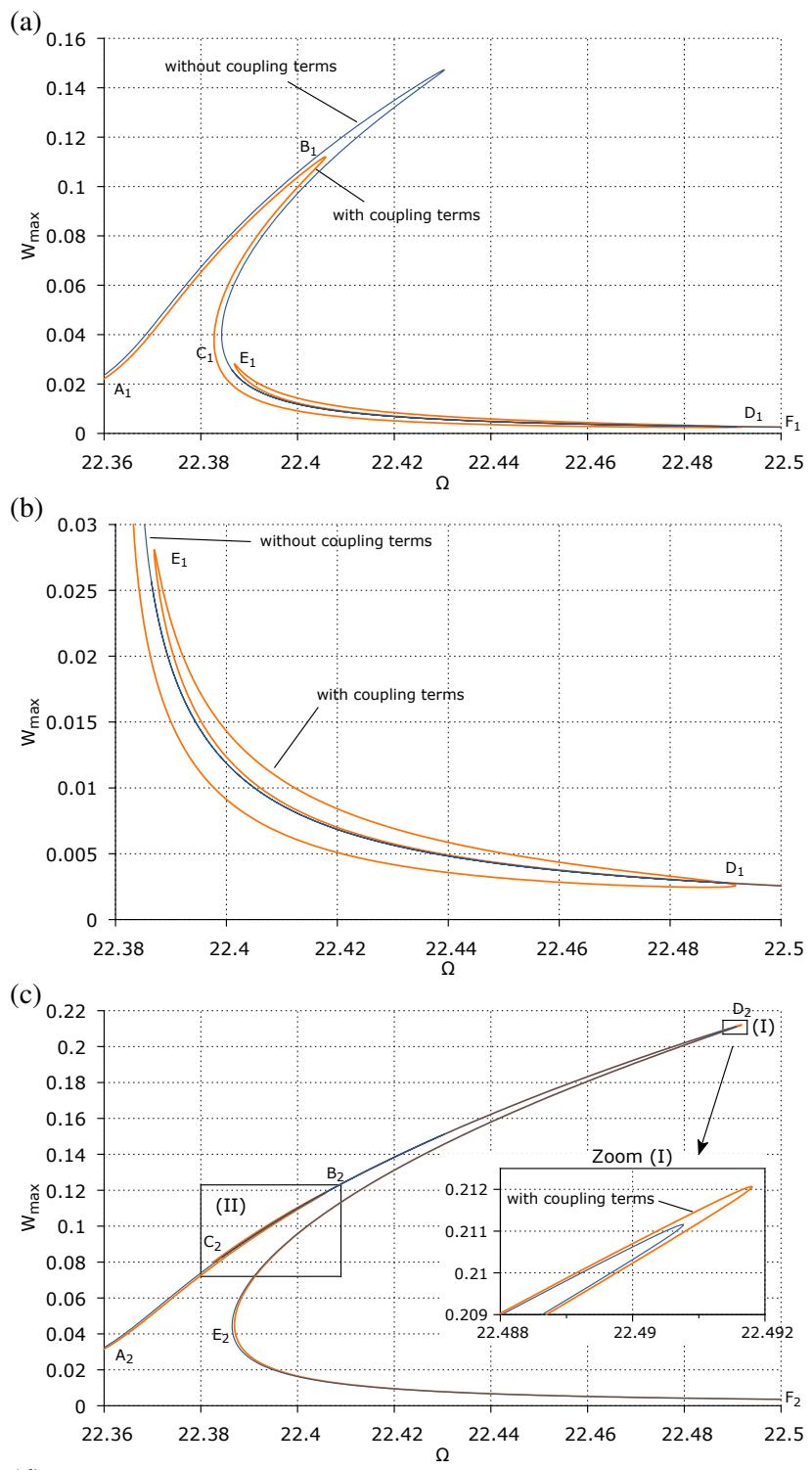

(d)

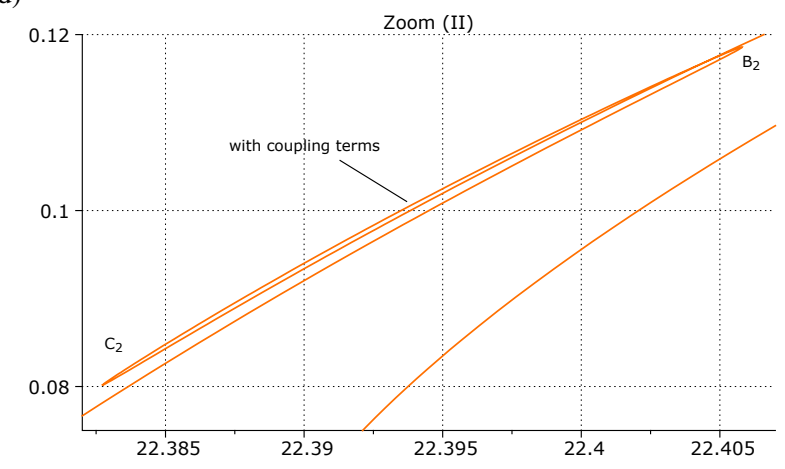

Fig. 4 Design \#1 without added mass. Response by neglecting the coupling terms (blue), complete response (orange). (a)-(b): Beam \#1, (c)(d): Beam \#2. 

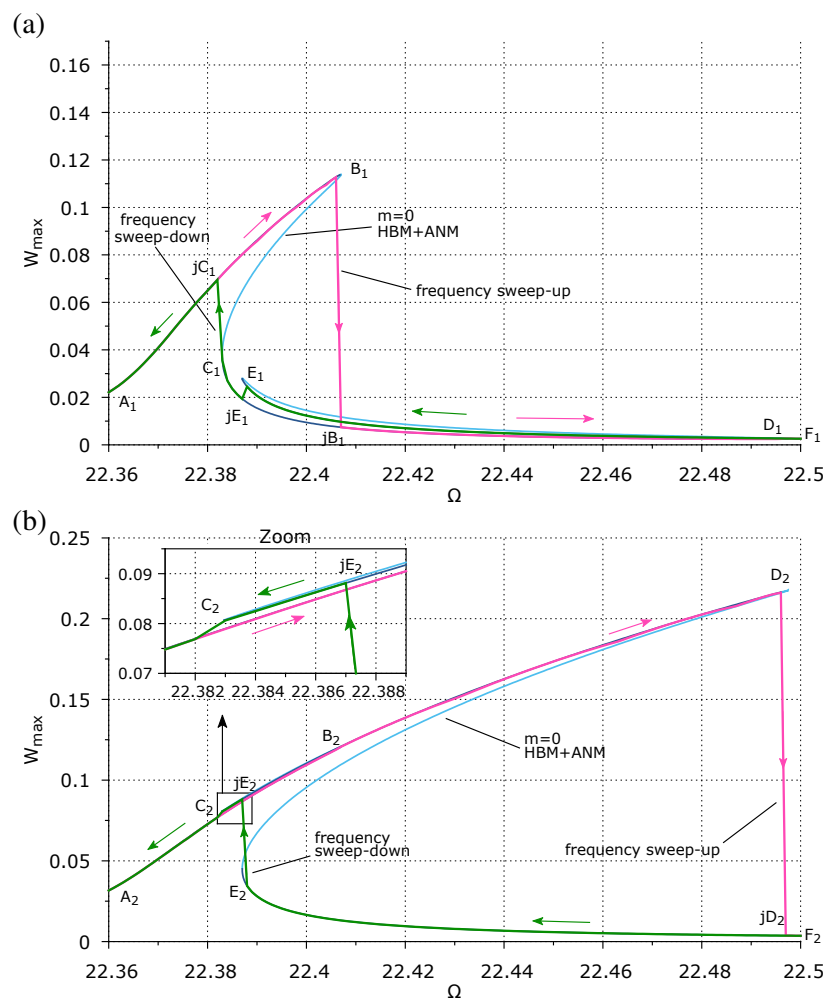

Fig. 5 Design \#1. Comparison between HBM+ANM (dark blue: stable branches, light blue: unstable branches) and time integration method with a frequency sweep-up (pink curves) and a frequency sweep-down (green curves). (a): Beam \#1, (b): Beam \#2.

stable branch $A_{2}-B_{2}-D_{2}-j D_{2}-F_{2}$ obtained by a frequency sweep-up but on the stable part of the $B_{2}-C_{2}$ loop.

295 Therefore, the pseudo arc-length continuation used in the $\mathrm{HBM}+\mathrm{ANM}$ method proves its efficiency and robustness here since it permits obtaining the complete response curve.

\subsection{Detection method}

300 In this section, a mass sensing method is developed. It is based on the exploitation of hysteresis cycles presented in $[12,31]$ in the case of a single resonator and extended here to the case of two-beam array.

Let the added mass fall on the second beam at $x_{0}=0.5$.

305 Fig. 6 presents the responses of the first and second beams with and without an added mass $m=10^{-4}$ determined by the HBM+ANM. The stable/unstable parts of the responses are plotted for $m=0$ and $m=10^{-4}$. Fig. 7 presents the response curves for the case of an added mass $m=10^{-4}$. It permits the

310 comparison between the results obtained by HBM+ANM and by time integration method with a frequency sweep-up and sweep-down.

From Fig. 6, it appears that the added mass makes the response more complicated due to the presence of several ad315 ditional loops. For the second beam, the amplitude is almost
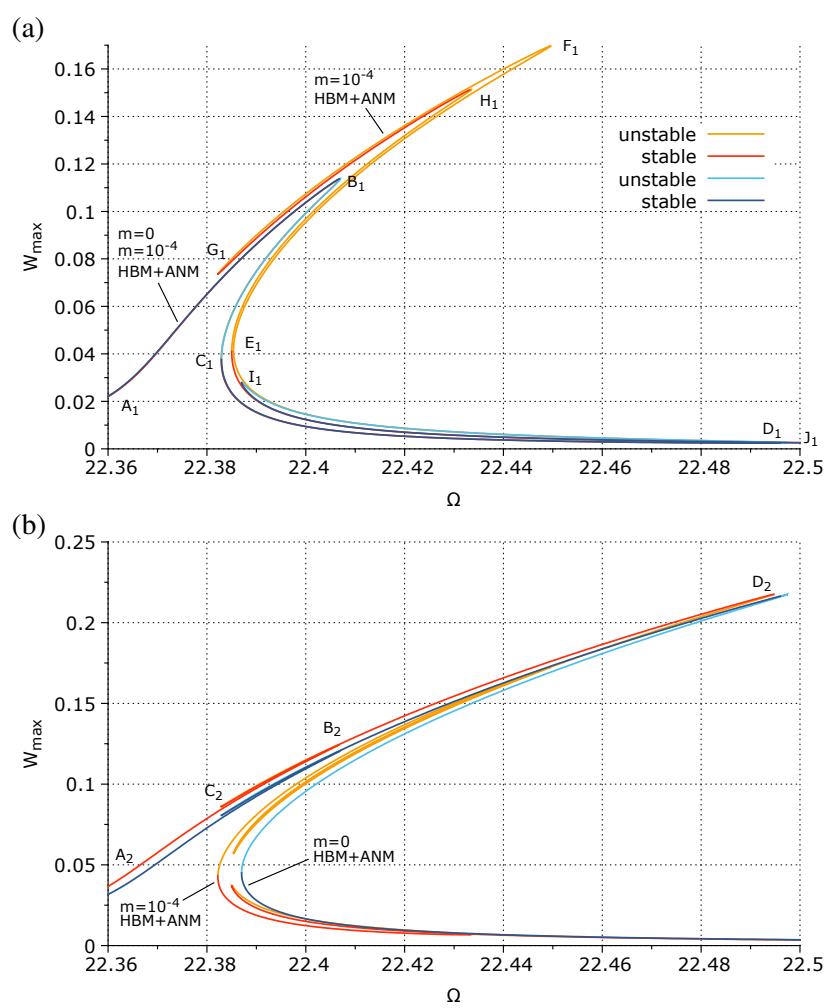

Fig. 6 Design \#1. Responses determined by the HBM+ANM method with an added mass $m=10^{-4}$ (red: stable branches, orange: unstable branches) and without added mass (dark blue: stable branches, light blue: unstable branches). (a): Beam \#1, (b): Beam \#2.

unchanged with an added mass $\left(W_{\max }=0.22\right)$ and there is a small frequency shift, while the first-beam amplitude changes from $W_{\max }\left(B_{1}\right) \approx 0.11$ to $W_{\max }\left(F_{1}\right) \approx 0.17$. With the aim of carrying out an experimental investigation, it is necessary 320 to verify, using the time integration method, that such an amplitude difference is detectable. In Fig. 7-(a), it can be observed that a simple frequency sweep-up or sweep-down does not permit the detection of the added mass. Indeed, the sweep-up and sweep-down responses coincide with the sta25 ble part of the response curve for $m=0$ of Fig. 6-(a) and the branches up to $H_{1}$ and $F_{1}$ are not obtained. The only difference concerns the position of the amplitude jump. During sweep-down, for the first beam, the jump occurs at $C_{1}$ without added mass and at $I_{1}$ with added mass. For the second beam, it occurs at $H_{2}$ and $G_{2}$ respectively. However, these differences are very difficult to distinguish for a small added mass.

To detect the added mass by means of amplitude difference, let both frequency sweeps be combined, see Fig. 8 . First, the frequency is swept down from point $J$ to a frequency value $\Omega_{\min }$ which is set between $\Omega\left(C_{1}\right)$ and $\Omega\left(I_{1}\right)$, then increased.

When using the time integration method, the response of the first beam is $J_{1}-i_{1}-j i_{1}-C_{1}-J_{1}\left(W_{\max }=0.02\right)$ without added mass and $J_{1}-I_{1}-j I_{1}-G_{1}-H_{1}-j H_{1}-J_{1}\left(W_{\max }=\right.$ 
(a)

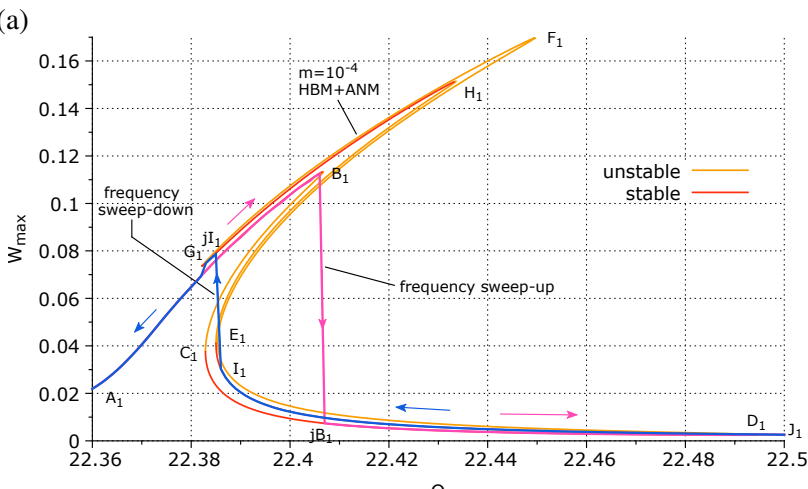

(b)

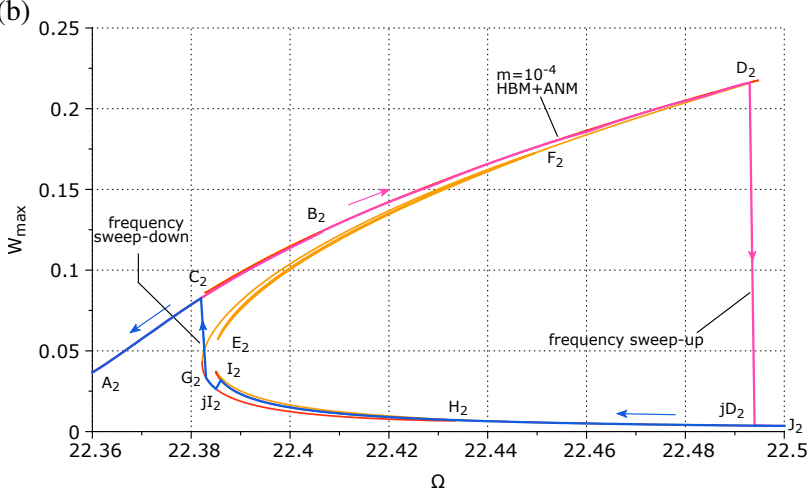

Fig. 7 Design \#1. Responses with added mass $m=10^{-4}$. Comparison between HBM+ANM (red: stable branches, orange: unstable branches) and time integration method with a frequency sweep-up (pink curves) and a frequency sweep-down (blue curves). (a): Beam \#1, (b): Beam $\# 2$.

$0.15)$ for $m=10^{-4}$. The response of the second beam is $J_{2}-$ $i_{2}-j i_{2}-g_{2}-d_{2}-j d_{2}-J_{2}\left(W_{\max }=0.22\right)$ without added mass and $J_{2}-I_{2}-j I_{2}-G_{2}-J_{2}\left(W_{\max }=0.04\right)$ for $m=10^{-4}$. Hence, the added mass is clearly detected by the difference 345 of amplitude.

If the mass $m=10^{-4}$ is added on the first beam instead of the second one, the beam-array response is almost unchanged because, as considered in the previous section, the influence of the second beam on the first beam is stronger 350 than the influence of the first beam on the second beam. Hence, a perturbation on the second-beam will cause a larger amplitude variation than a perturbation on the first beam.

The threshold of detection can be set by adjusting voltages. In order to adjust the mass threshold, $\Omega_{A_{1}}$ has to be su355 perior to $\Omega_{A_{2} m}$ and inferior to $\Omega_{A_{2}}$, see Fig.9. For example, when decreasing $V_{d c_{32}}$ and $V_{a c_{32}}$ to $0.575 \mathrm{~V}$ instead of $0.6 \mathrm{~V}$ while keeping other voltages unchanged, see Table $1, \Omega_{A_{2}}$ becomes closer to $\Omega_{A_{1}}$. Therefore, smaller masses, such as $m=10^{-5}$, can also be detected by a large amplitude change.

360 For a bigger mass, such as $m=10^{-3}$, the second beam response has a lower amplitude, see Fig. 10. By adding a mass bigger than an upper threshold, $\Omega_{B_{2} m}$ becomes larger than $\Omega_{B_{1} m}$ and the amplitude of the second beam response decreases. Therefore, larger masses such as $m=10^{-3}$ can this
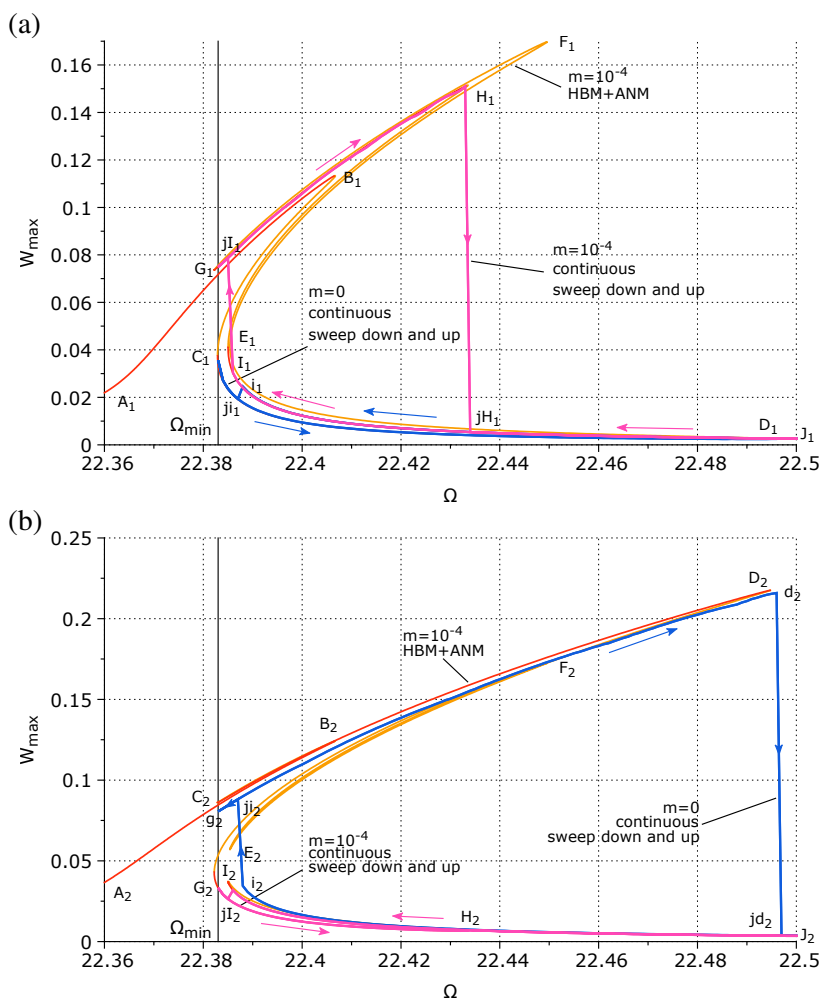

Fig. 8 Design \#1. Responses determined by time integration method without added mass (blue curves $J_{1}-i_{1}-j i_{1}-C_{1}-J_{1}$ and $J_{2}-i_{2}-$ $j i_{2}-g_{2}-d_{2}-j d_{2}-J_{2}$ ) and with added mass $m=10^{-4}$ (green curves $J_{1}-I_{1}-j I_{1}-G_{1}-H_{1}-j H_{1}-J_{1}$ and $\left.J_{2}-I_{2}-j I_{2}-G_{2}-J_{2}\right)$; responses determined by HBM+ANM with added mass $m=10^{-4}$ (red: stable branches, orange: unstable branches). (a): Beam \#1, (b): Beam \#2.

365 time be detected by a reduced amplitude on the second beam response curve. To sum up, there exist two mass thresholds. When the first one is exceeded there is a large amplitude jump on the first beam amplitude. Then, when the added mass is higher than the second threshold, the amplitude of 70 the second beam starts to decrease. But for added masses between these two thresholds, the responses are not so different.

\section{Three-beam array}

In order to overcome the limitations of the sensing method 35 presented in the previous section, an alternative method based on symmetry breaking is considered in this section.

An array of three identical beams sketched in Fig. 11 is now considered, the beam dimensions and all parameters being similar to those considered in the previous section. Let 30 the cases of asymmetric and symmetric voltages be distinguished. Asymmetric voltages lead to results very similar to the two-beam array. On the response curves, there are two small loops due to the influence of the two other beams, see Fig.19 in Appendix C. In the case of symmetric voltages, 


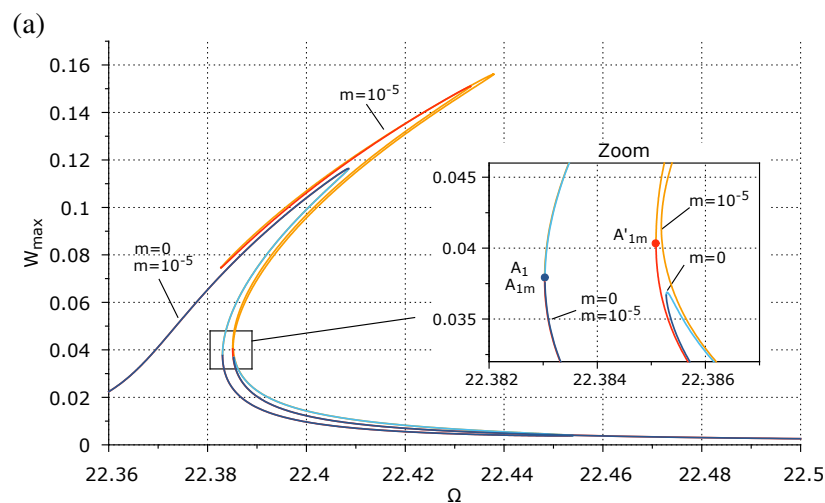

(b)

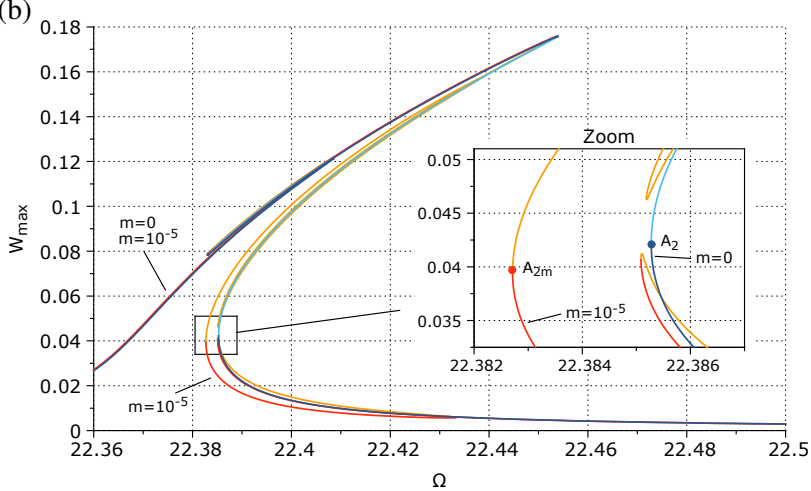

Fig. 9 Response with $V_{d c_{32}}=V_{a c_{32}}=0.575 \mathrm{~V}$ and other voltages as in Table 1, without mass (dark/light blue) and with $m=10^{-5}$ (red/orange). (a): Beam \#1, (b): Beam \#2.
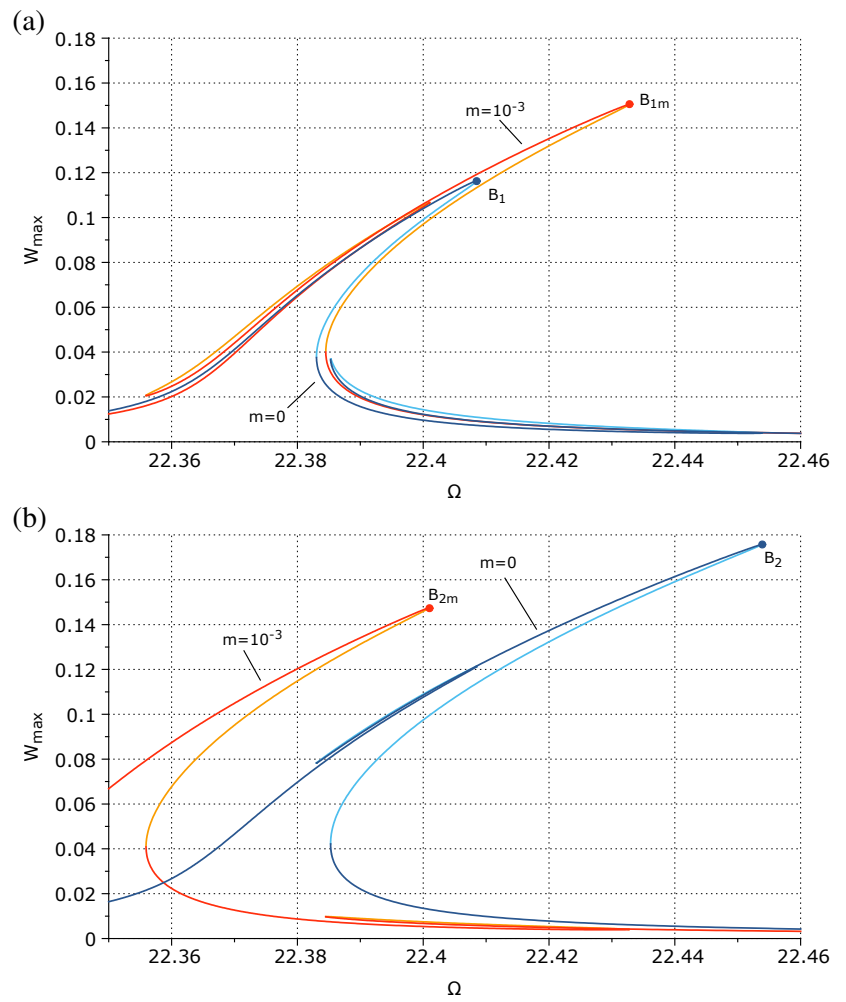

Fig. 10 Response with $V_{d c_{32}}=V_{a c_{32}}=0.575 \mathrm{~V}$ and other voltages as in Table 1, without mass (dark/light blue) and with $m=10^{-3}$ (red/orange). (a): Beam \#1, (b): Beam \#2.

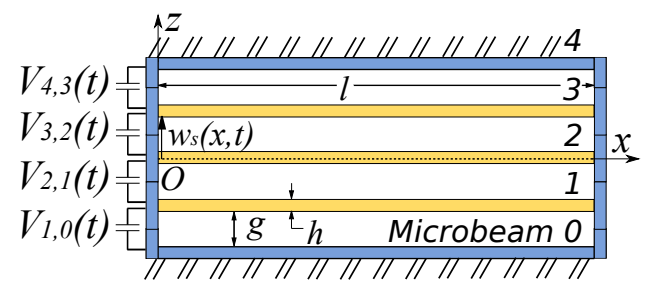

Fig. 11 Array of three clamped-clamped microbeams.

385 designs with small (Design \#2) and large (Designs \#3 and \#4) voltages are investigated with values presented in Table 2.

Table 2 Symmetric actuation voltages of the 3-beam array.

\begin{tabular}{|c|c|c|c|c|}
\hline Design & $V d c_{10}(V)$ & $V d c_{21}(V)$ & $V d c_{32}(V)$ & $V d c_{43}(V)$ \\
& $V a c_{10}(V)$ & $V a c_{21}(V)$ & $V a c_{32}(V)$ & $V a c_{43}(V)$ \\
\hline$\# 2$ & 0 & 0.28 & 0.28 & 0 \\
& 0 & 0.88 & 0.88 & 0 \\
\hline$\# 3$ & 0 & 5 & 5 & 0 \\
& 0 & 1 & 1 & 0 \\
\hline$\# 4$ & 0 & 5.3 & 5.3 & 0 \\
& 0 & 1 & 1 & 0 \\
\hline
\end{tabular}

\subsection{Design \#2}

Due to the symmetric voltages, the electrostatic forces on 390 both sides of the second beam are balanced. Hence the second beam does not vibrate. If the added mass falls on it, the system is still symmetric and the second beam remains at rest. If the added mass falls on the first or third beam, the system loses its symmetry and the response changes. How395 ever, depending on the value of added mass, the response change for the first and third beams can be very small and difficult to identify. This is why the detection is based on the second-beam response instead.

Figure 12 shows the case of an added mass $m=10^{-3}$ at 400 the middle of beam \#1 $\left(x_{0}=0.5\right)$. Without added mass, the responses of beams \#1 and \#3 are identical. With an added mass, the response of beam \#1 is shifted to the left and loops appear on the responses of beams \#1 and \#3. However, the response amplitudes do not change in comparison with the 405 case without added mass. The amplitude of beam \#2 is nil $\left(W_{\max }=0\right)$ without added mass but large with $m=10^{-3}$ as a result of symmetry breaking.

When voltages $V a c_{21}$ and $V d c_{21}$ between beams \#1 and $\# 2$ are identical to voltages $V a c_{32}$ and $V c d_{32}$ between beams \#2 and \#3, the electrostatic forces and the second-beam displacement depend only on the difference between the deflections of beams \#1 and \#3. The larger the added mass is, 


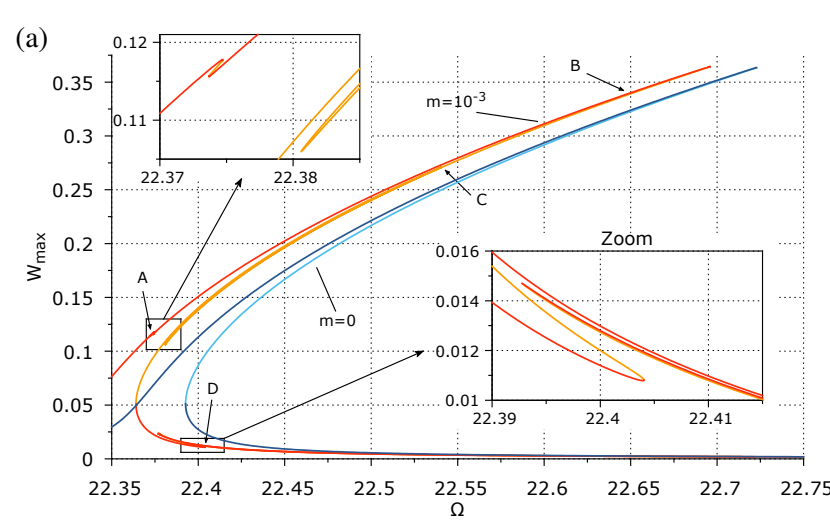

(b)

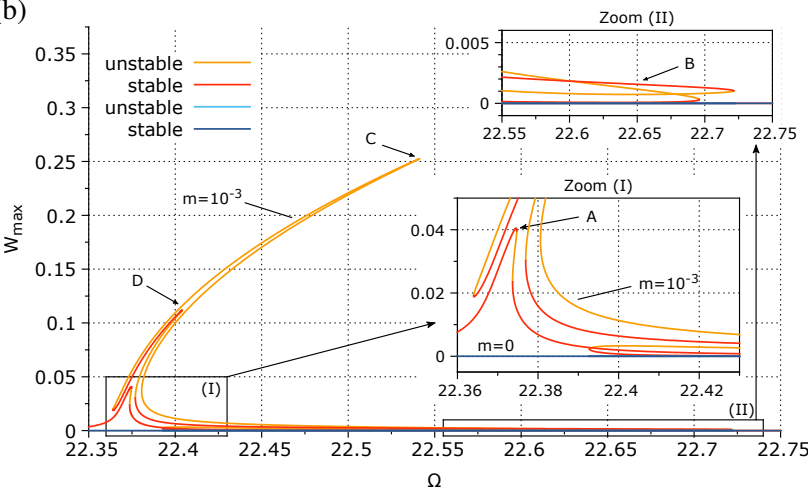

(c)

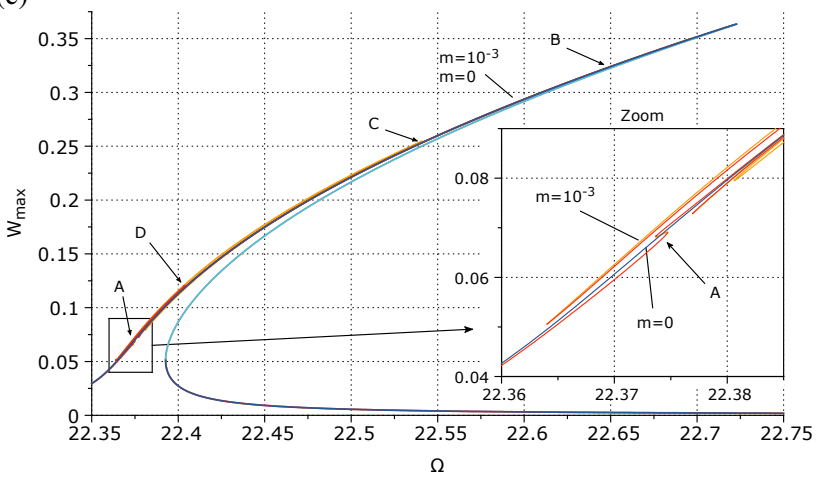

Fig. 12 Design \#2: response with a mass $m=10^{-3}$ added on the first beam (red/orange) and without added mass (dark/light blue). (a): Beam \#1, (b): Beam \#2, (c): Beam \#3. Without added mass, the second-beam amplitude is nil.

the more different these displacements are and the larger the second-beam displacement is. This is illustrated in Fig. 13 415 showing the second-beam response for several values of the added mass.

With a mass $m=10^{-3}$ added on beam \#1, the deflections of beams \#1 and \#3 are different. Let points $A, B, C, D$ be considered as defined in Figs. 12 and 13-(a). At these points, 420 the displacements of beams \#1 and \#3 are plotted in Fig. 13(b). For each value of time $t$, these displacements are the most different at point $\mathrm{C}$ and the less different at point $\mathrm{B}$. Therefore, it results that $W_{C}>W_{D}>W_{A}>W_{B}$ as observed in Fig. 13-(a).

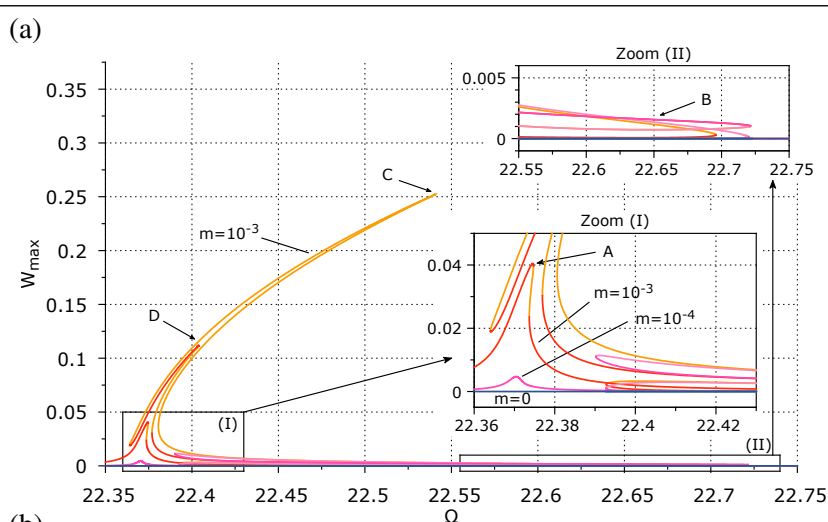

(b)

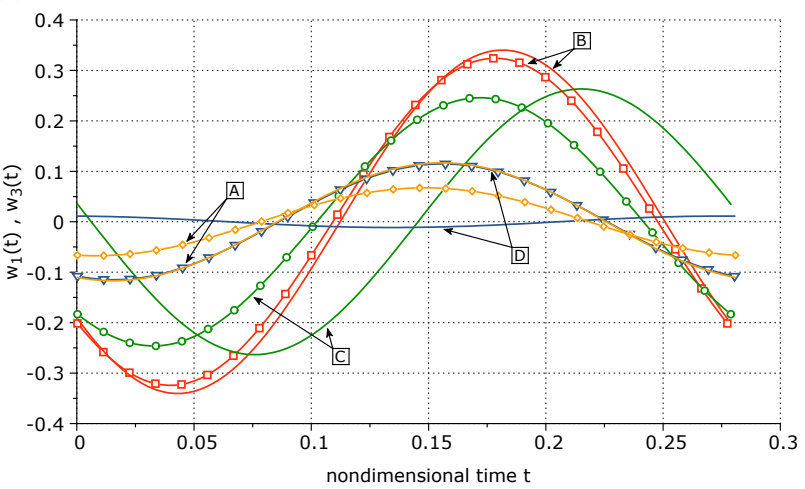

Fig. 13 Design \#2: (a): response of Beam \#2 without (blue curve) and with $m=10^{-4}$ (pink curve) or $m=10^{-3}$ (red/orange curve) added on Beam \#1. (b): displacement of Beam \#1 (solid line) and Beam \#3 (dotted line) at points A (yellow curves), B (red curves), C (green curves) and $\mathrm{D}$ (blue curves) with $m=10^{-3}$.

The amplitude of Beam \#2 is large, with $W_{\max }=0.25$ at point $\mathrm{C}$. However, this branch is unstable, thus not observable experimentally. Fig. 14 represents the responses obtained by time integration with a frequency sweep-up and sweep-down. As for the two-beam system, these responses are not coincident. As shown in Fig. 14-(a), with a frequency sweep-up the maximum amplitude $W_{\max }=0.04$ is reached just before the jump down to point $\mathrm{E}$ and $W_{\max }=0.04$ whereas, with a sweep down, the maximum amplitude is $W_{\max }=0.05$ at point $\mathrm{F}$.

A larger amplitude can be reached if the two frequency sweeps are combined as follows (see Fig. 14-(b)): first a sweep-down until the jump at point $F$, then a sweep-up, resulting in a maximum amplitude $W_{\max }=0.11$ at point $\mathrm{D}$ that can be easily detected.

However, with a smaller added mass, e.g. $m=10^{-4}$, the difference between the displacements of Beams \#1 and \#3 is small. Thus the deflection of Beam \#2 is also small, as shown in Fig. 13-(a), making the detection difficult. As explained in Section 3.4 there is a threshold of added mass, above which additional loops increase the response amplitude. Therefore the added mass must be larger than the threshold for the symmetry breaking to generate a high amplitude 


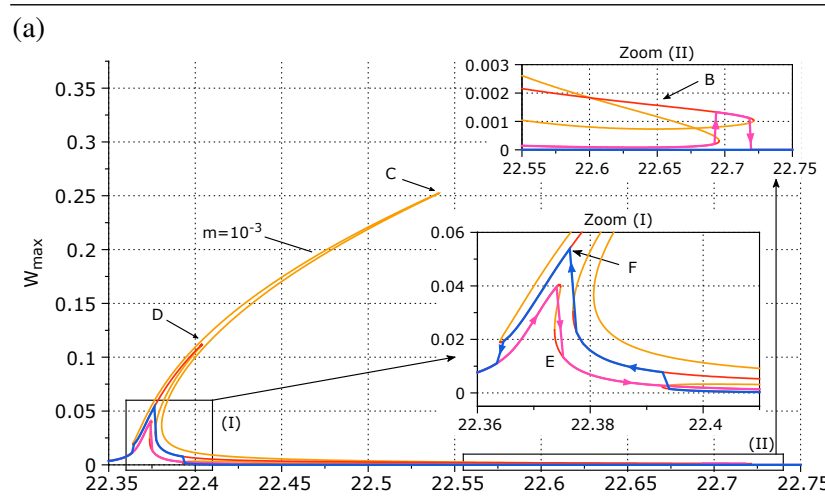

(b)

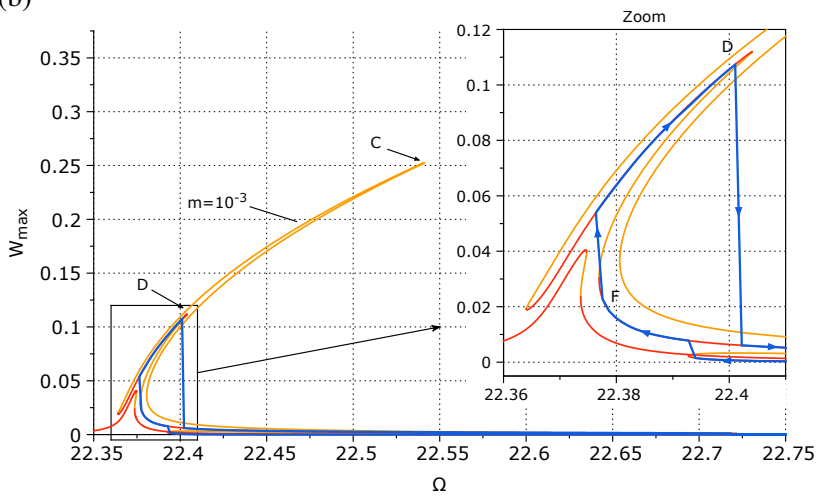

Fig. 14 Design \#2. Response of Beam \#2 with $m=10^{-3}$ added on Beam \#1. (a): HBM+ANM (red/orange curve), time integration with a sweep-up (pink curve) and a sweep-down (blue curve); (b): $\mathrm{HBM}+\mathrm{ANM}$ (red/orange curve), time integration method combining a frequency sweep-down then sweep-up (blue curve).

on Beam \#2. To detect smaller added masses, let Design \#3 be considered.

\section{4.2 Designs \#3 and \#4}

This section considers the case of large symmetric voltages, with Designs \#3 and \#4 given in Table 2. Since large voltages generate large vibration amplitudes, considering the response robustness is necessary.

455 The responses for Design \#3 are presented in Fig. 15. The considered voltages are large enough for a frequency sweep-up from $\Omega=22.35$ to $\Omega=22.75$ to make the resonator collapse by pull-in $\left(W_{\max } \geq 1\right)$. Hence, the sweep-up is performed only in the interval $\Omega=[22.17-22.23]$ where ${ }_{460}$ the resonator has not yet collapsed. Without added mass, the calculation with one mode and a third-order Taylor series shows convergent responses. The array remains symmetric, so beam \#2 does not vibrate.

With a mass $m=10^{-4}$ added on the first beam, because 465 of the symmetry-breaking, the second-beam amplitude is large. The calculations have been limited to the first three modes and use a seventh-order Taylor series in order to keep an affordable computational time. In Fig. 15, with the same order of Taylor series $(T=3,5$ or 7$)$, the responses with
Table 3 Correspondence between the non dimensional mass ratio $m$ and the physical value of the added mass $m_{p}$

\begin{tabular}{|c|c|c|c|}
\hline$m$ & $10^{-3}$ & $10^{-4}$ & $5 \times 10^{-4}$ \\
\hline$m_{p}(k g)$ & $1.1184^{-18}$ & $1.1184^{-19}$ & $2.2368^{-20}$ \\
\hline \hline$m$ & $10^{-6}$ & $10^{-8}$ & \\
\hline$m_{p}(k g)$ & $1.1184^{-21}$ & $1.1184^{-23}$ & \\
\hline
\end{tabular}

${ }_{470} N_{m}=1$ or $N_{m}=2$ modes are almost unchanged. On the contrary, with a fixed number of modes, the responses change a lot when using the third or fifth order, while the seventh order does not improve the solution anymore. For smaller added masses, the influence of high orders is more signifi475 cant.

Figure 16 shows the responses for Design \#4 (slightly higher symmetric voltage, see Table 2), calculated with $N_{m}=$ 1 mode. Using third-order Taylor series, an added mass $m=$ $10^{-6}$ can be detected by a small difference between the re480 sponses with and without added mass. However, with seventhorder series, a smaller added mass $m=10^{-12}$ can be detected because a larger difference is observed. Theoretically, any infinitesimal perturbation causes the symmetry-breaking of the three-beam array. However, from a numerical point of 85 view, the detection threshold depends on the accuracy of the model. Thus, the detection threshold can be improved by increasing the order of the Taylor series. It is however limited by the computational cost and the numerical accuracy of the algorithms.

490 Fig. 17 presents the responses calculated with $N_{m}=1$ and seventh-order Taylor series for increasing values of $m$. Without added mass, the amplitude of beam \#2 is $W_{\max }=0$ and it changes from $W_{\max }=0.05$ for $m=10^{-12}$ (green) to $W_{\text {max }}=0.2$ for $m=5 \times 10^{-4}$ (cyan). For large values of ${ }_{495} m\left(m>10^{-6}\right)$, the quantification is possible. However, for small values of $m\left(m<10^{-6}\right)$ the difference in amplitude is very small so an accurate quantification is difficult, see Fig. 18. Once the quantification of the non-dimensional ratio $m$ is made, the corresponding physical added mass $m_{p}$ is 500 obtained with Eq. (19) as listed in Table 3 for a few values of $m$.

It is worth mentioning that the sensing method presented in this section can only be applied if the beam array is perfectly symmetric. This is not the case of fabricated devices 505 which inevitably contain manufacturing defects. Nevertheless, as shown in [32], it is possible to compensate the manufacturing defects of one beam by tuning the electrostatic actuation of adjacent beams, thus turning an imperfect device into a perfect one. In order to evaluate the required level of 10 tuning, the measured fabrication errors could be introduced in the model in similar way as in [33]. 

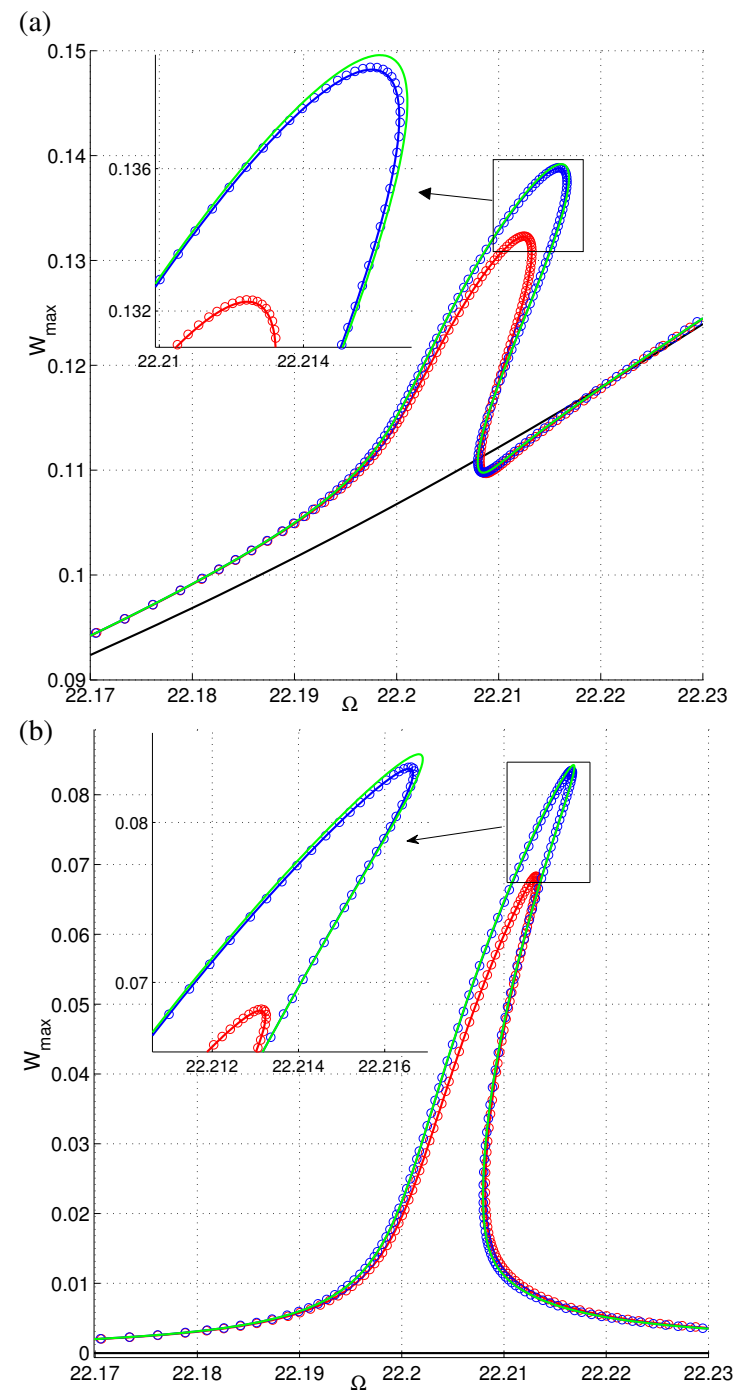

(c)

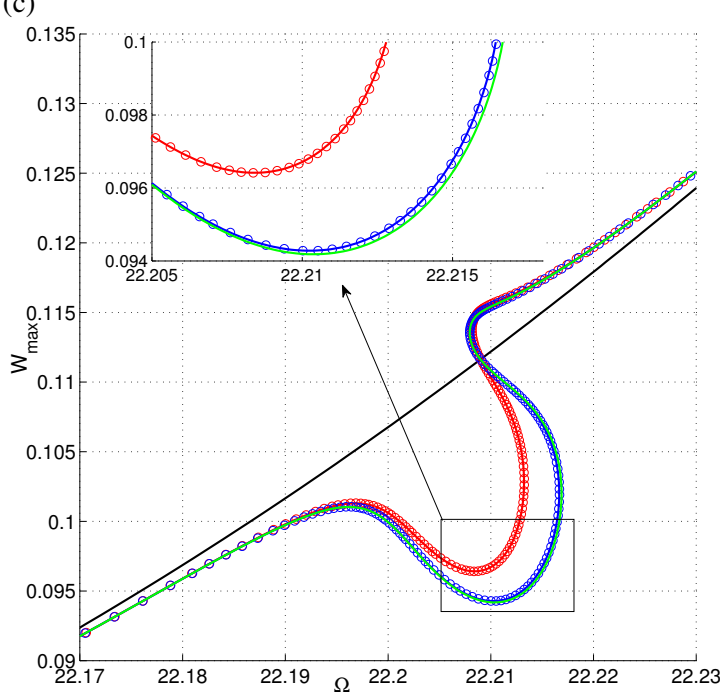

Fig. 15 Design \#3, responses with an added mass $m=10^{-4}$ on the first beam determined by using third (red), fifth (blue) and seventh (green) order Taylor series with $N_{m}=1$ (solid line) or $N_{m}=2$ (circle) modes; responses without added mass (black). (a): Beam \#1, (b): Beam \#2, (c): Beam \#3.
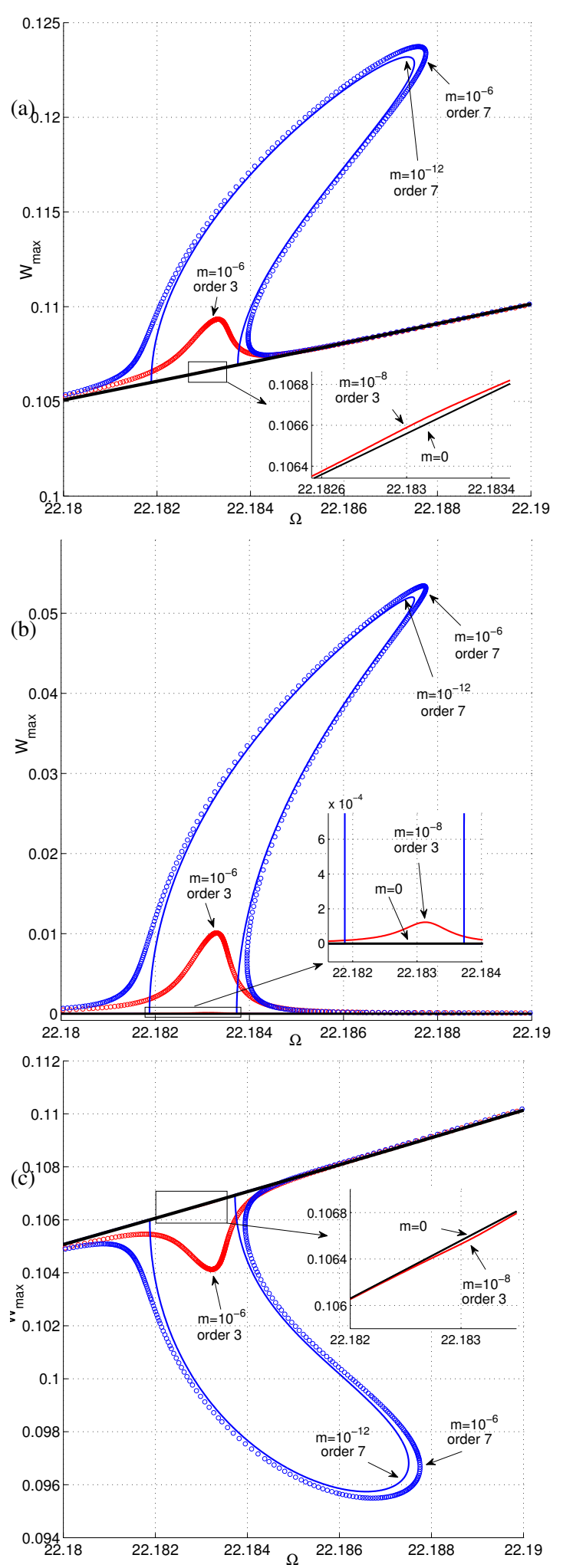

Fig. 16 Design \#4, responses with $N_{m}=1$ mode. Red curves: responses with $m=10^{-8}$ (solid line) or $m=10^{-6}$ (circle) on the first beam determined by third-order Taylor series. Blue curves: responses with $m=10^{-12}$ (solid line) or $m=10^{-6}$ (circle) on the first beam determined by seventh-order Taylor series. Black curves: responses without added mass. (a): Beam \#1, (b): Beam \#2, (c): Beam \#3. 

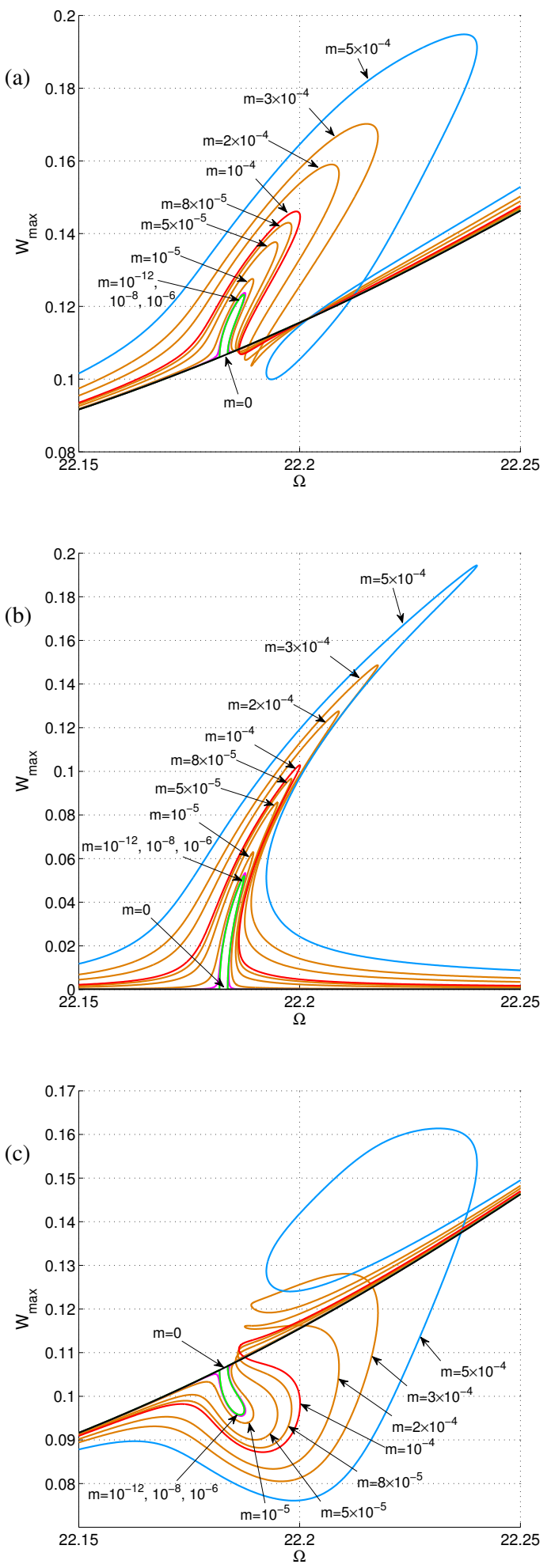

Fig. 17 Design \#4. Responses with $N_{m}=1$ mode and seventh order Taylor series, without added mass (black), with $m=10^{-12}$ (green), $m=10^{-6}$ (magenta), $m=10^{-4}$ (red), $m=5 \times 10^{-4}$ (blue) added on the first beam. (a): Beam \#1, (b): Beam \#2, (c): Beam \#3.

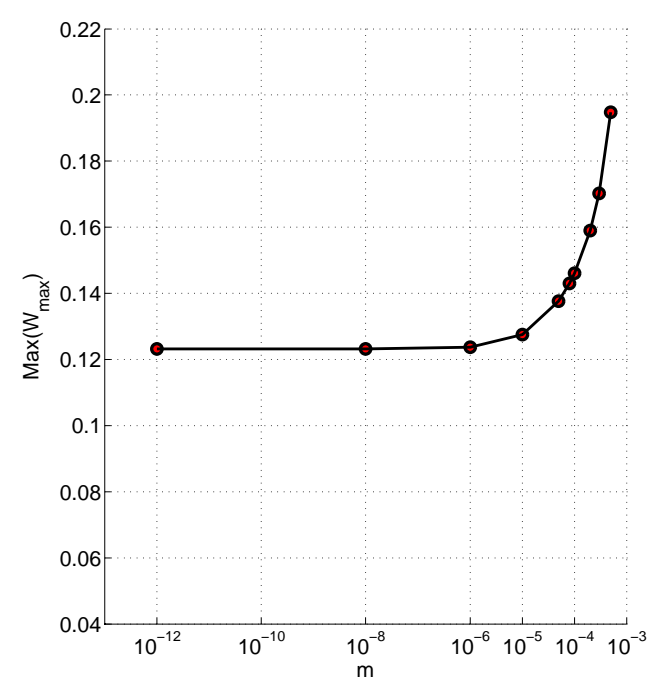

Fig. 18 Design \#4. Maximum of amplitude $\operatorname{Max}\left(W_{\max }\right)$ with respect to added mass $m$ for Beam \#2.

\section{Conclusion}

In this paper, the fundamental resonance of an array of identical microbeams is considered. HBM+ANM, time integra515 tion and averaging methods are used to determine the responses. The robustness of the ANM-based continuation enables to follow very complex responses with stable and unstable branches. The time integration method is used to simulate experimental results and the averaging method per520 mits analyzing the influence of adjacent beams. Because of this influence, each beam response presents a very complicated nonlinear behavior with several loops, the influence of distant beams being weaker. Results based on time integration show that the combination of two frequency sweeps ${ }_{525}$ (down then up) is necessary for experimental detection of an added mass. For an asymmetric beam array, a small added mass can be detected by tuning voltages to a threshold. In particular, in symmetric configurations, the mass detection can be highly improved by using bifurcations of symmetry530 breaking type.

Acknowledgements The authors are indebted to the Institute Carnot Ingénierie@Lyon for its support and funding of the NEMROD project.

Conflict of Interest The authors declare that they have no conflict of interest.

\section{${ }_{535}$ References}

1. N. Kacem, S. Baguet, S. Hentz, and R. Dufour. Nonlinear phenomena in nanomechanical resonators: mechanical behaviors and physical limitations. Mec. Ind., 11(6):521-529, 2010.

2. M. Saghafi, H. Dankowicz, and W. Lacarbonara. Nonlinear tuning of microresonators for dynamic range enhancement. Proc. R. Soc. London, Ser. A, 471(2179):20140969, 2015. 
3. M.I. Younis. MEMS Linear and Nonlinear Statics and Dynamics. 610 23. S. Gutschmidt and O. Gottlieb. Nonlinear internal resonances of Springer, 2011.

4. M. Narducci, E. Figueras, M.J. Lopez, I. Gracia, J. Santander, P. Ivanov, L. Fonseca, and C. Cané. Sensitivity improvement of a microcantilever based mass sensor. Microelectron. Eng., 86(46): $1187-1189,2009$.

5. S. Dohn, R. Sandberg, W. Svendsen, and A. Boisen. Enhanced functionality of cantilever based mass sensors using higher modes and functionalized particles. In Solid-State Sensors, Actuators and Microsystems TRANSDUCERS '05., volume 1, pages 636-639, 2005.

6. H. Xie, J. Vitard, S. Haliyo, and S. Régnier. Enhanced sensitivity of mass detection using the first torsional mode of microcantilevers. Meas. Sci. Technol., 19(5):055207, 2008.

7. A. Eichler, J. Moser, M.I. Dykman, and A. Bachtold. Symmetry breaking in a mechanical resonator made from a carbon nanotube. Nat. Commun., 4(2843), 2013.

8. J.F. Rhoads, S.W. Shaw, and K.L. Turner. Nonlinear dynamics and its applications in micro- and nanoresonators. J. Dyn. Syst. Meas. Contr., 132(3):034001, 2010.

9. M.I. Younis and F. Alsaleem. Exploration of new concepts for mass detection in electrostatically-actuated structures based on nonlinear phenomena. J. Comput. Nonlinear Dyn., 4(2):021010, 2009.

10. L. Ruzziconi, S. Lenci, and M.I. Younis. An imperfect microbeam under an axial load and electric excitation: nonlinear phenomena and dynamical integrity. Int. J. Bifurcation Chaos, 23(02):1350026, 2013.

57011 . V. Kumar, J. W. Boley, Y. Yang, H. Ekowaluyo, J. K. Miller, G. T.C. Chiu, and J. F. Rhoads. Bifurcation-based mass sensing using piezoelectrically-actuated microcantilevers. Appl. Phys. Lett., 98(15):153510, 2011.

12. V.-N. Nguyen, S. Baguet, C.-H. Lamarque, and R. Dufour. 575 Bifurcation-based micro-/nano-electromechanical mass detection. Nonlinear Dyn., 79(1):647-662, 2014.

13. W. Zhang and K.L. Turner. Application of parametric resonance amplification in a single-crystal silicon micro-oscillator based ${ }^{645}$ mass sensor. Sens. Actuators, A, 122(1):23-30, 2005.

580 14. O. Thomas, F. Mathieu, W. Mansfield, C. Huang, S. TrolierMcKinstry, and L. Nicu. Efficient parametric amplification in micro-resonators with integrated piezoelectric actuation and sensing capabilities. Appl. Phys. Lett., 102(16):163504, 2013.

15. N. Kacem, S. Baguet, L. Duraffourg, G. Jourdan, R. Dufour, and

585 S. Hentz. Overcoming limitations of nanomechanical resonators with simultaneous resonances. Appl. Phys. Lett., 107(7):073105, 2015.

16. H. B. Peng, C. W. Chang, S. Aloni, T. D. Yuzvinsky, and A. Zettl. Ultrahigh frequency nanotube resonators. Phys. Rev. Lett., 97:087203, 2006.

17. M.S. Hanay, S. Kelber, A.K. Naik, D. Chi, S. Hentz, E.C. Bullard, E. Colinet, L. Duraffourg, and M.L. Roukes. Single-protein nanomechanical mass spectrometry in real time. Nat. Nanotechnol., 7:602-608, 2012.

595 18. E. Buks and M. L. Roukes. Electrically tunable collective response in a coupled micromechanical array. J. Microelectromech. Syst., 11(6):802-807, 2002.

19. R. Lifshitz and M. C. Cross. Response of parametrically driven nonlinear coupled oscillators with application to micromechanical and nanomechanical resonator arrays. Phys. Rev. B, 67:134302, 2003.

20. M. C. Cross, A. Zumdieck, R. Lifshitz, and J. L. Rogers. Synchronization by nonlinear frequency pulling. Phys. Rev. Lett., 93:224101, 2004

605 21. M. Porfiri. Vibrations of parallel arrays of electrostatically actuated microplates. J. Sound Vib., 315(4):1071-1085, 2008.

22. R. B. Karabalin, M. C. Cross, and M. L. Roukes. Nonlinear dynamics and chaos in two coupled nanomechanical resonators. Phys. Rev. B, 79:165309, 2009.

a microbeam array near the pull-in point. In Proc. ENOC-2008, Saint Petersburg, June, 30 -July, 42008.

24. S. Gutschmidt and O. Gottlieb. Internal resonances and bifurcations of an array below the first pull-in instability. Int. J. Bifurcation Chaos, 20(3):605-618, 2010.

25. S. Gutschmidt and O. Gottlieb. Bifurcations and loss of orbital stability in nonlinear viscoelastic beam arrays subject to parametric actuation. J. Sound Vib., 329:3835-3855, 2010.

26. S. Gutschmidt and O. Gottlieb. Nonlinear dynamic behavior of a 620 microbeam array subject to parametric actuation at low, medium and large dc-voltages. Nonlinear Dyn., 67(1):1-36, 2012.

27. P.N. Kambali, G. Swain, A.K. Pandey, E. Buks, and O. Gottlieb. Coupling and tuning of modal frequencies in direct current biased microelectromechanical systems arrays. Appl. Phys. Lett., 107(6):063104, 2015.

28. M.I. Younis, E.M. Abdel-Rahman, and A. Nayfeh. A reducedorder model for electrically actuated microbeam-based MEMS. $J$. Microelectromech. Syst., 12(5):672-680, 2003.

29. N. Kacem, J. Arcamone, F. Perez-Murano, and S. Hentz. Dynamic range enhancement of nonlinear nanomechanical resonant cantilevers for highly sensitive nems gas/mass sensor applications. J. Micromech. Microeng., 20(4):045023, 2010.

30. N. Kacem, S. Baguet, S. Hentz, and R. Dufour. Computational and quasi-analytical models for non-linear vibrations of resonant MEMS and NEMS sensors. Int. J. Non Linear Mech., 46(3):532 $542,2011$.

31. M. Sansa, V.-N. Nguyen, S. Baguet, C.-H. Lamarque, R. Dufour, and S. Hentz. Real time sensing in the non linear regime of nems resonators. In 2016 IEEE 29th International Conference on Micro Electro Mechanical Systems (MEMS), pages 1050-1053, Jan., 24 282016.

32. V. Walter, G. Bourbon, P. Le Moal, N. Kacem, and J. Lardis. Electrostatic actuation to counterbalance the manufacturing defects in a mems mass detection sensor using mode localization. Procedia Eng., 168:1488-1491, 2016.

33. L. Ruzziconi, A. M. Bataineh, M. I. Younis, W. Cui, and S. Lenci. Nonlinear dynamics of an electrically actuated imperfect microbeam resonator: experimental investigation and reduced-order modeling. J. Micromech. Microeng., 23(7):075012, 2013.

\section{Appendix A Reduced-order model}

The non-dimensional equation of motion for beam $s$ is

$$
\begin{aligned}
\frac{\partial^{4} w_{s}}{\partial x^{4}}+\frac{\partial^{2} w_{s}}{\partial t^{2}} & +c \frac{\partial w_{s}}{\partial t}-\left[N+\alpha_{1} \int_{0}^{1}\left(\frac{\partial w}{\partial x}\right)^{2} \mathrm{~d} x\right] \frac{\partial^{2} w_{s}}{\partial x^{2}} \\
& =\alpha_{2} \frac{V_{s, s+1}^{2}}{\left(1+w_{s+1}-w_{s}\right)^{2}}-\alpha_{2} \frac{V_{s-1, s}^{2}}{\left(1+w_{s}-w_{s-1}\right)^{2}}
\end{aligned}
$$

By using the Galerkin method and seventh-order Taylor series Eq.28 is replaced by a set of equations in the following matrix form

$$
\begin{aligned}
& \ddot{\boldsymbol{a}}^{s}+\boldsymbol{C}_{0} \dot{\boldsymbol{a}}^{s}+\boldsymbol{K}_{0} \boldsymbol{a}^{s}-\left(N+\alpha_{1} T_{2}^{s}\left(\boldsymbol{a}^{s}\right)\right) \boldsymbol{K}_{T} \boldsymbol{a}^{s}= \\
& \alpha_{2} V_{s, s+1}^{2} \boldsymbol{Q}_{0}+\alpha_{2} V_{s, s+1}^{2}\left[\boldsymbol{Q}_{1}+\boldsymbol{Q}_{2}^{s}\left(\boldsymbol{b}^{s}\right)+\boldsymbol{Q}_{3}^{s}\left(\boldsymbol{b}^{s}\right)+\boldsymbol{Q}_{4}^{s}\left(\boldsymbol{b}^{s}\right)+\boldsymbol{Q}_{5}^{s}\left(\boldsymbol{b}^{s}\right)\right. \\
& \left.+\boldsymbol{Q}_{6}^{s}\left(\boldsymbol{b}^{s}\right)+\boldsymbol{Q}_{7}^{s}\left(\boldsymbol{b}^{s}\right)\right] \boldsymbol{b}^{s}-\alpha_{2} V_{s-1, s} V_{s-1, s}^{2} \boldsymbol{P}_{0} \\
& -\alpha_{2} V_{s-1, s}^{2}\left[\boldsymbol{P}_{1}+\boldsymbol{P}_{2}^{s}\left(\boldsymbol{b}^{s-1}\right)+\boldsymbol{P}_{3}^{s}\left(\boldsymbol{b}^{s-1}\right)\right. \\
& \left.\quad+\boldsymbol{P}_{4}^{s}\left(\boldsymbol{b}^{s-1}\right)+\boldsymbol{P}_{5}^{s}\left(\boldsymbol{b}^{s-1}\right)+\boldsymbol{P}_{6}^{s}\left(\boldsymbol{b}^{s-1}\right)+\boldsymbol{P}_{7}^{s}\left(\boldsymbol{b}^{s-1}\right)\right] \boldsymbol{b}^{s-1}
\end{aligned}
$$


The components of the matrices are given by

$$
\begin{aligned}
& \boldsymbol{C}_{0 i j}=c_{i} \delta_{i j}, \quad \boldsymbol{K}_{0 i j}=\lambda_{i}^{4} \delta_{i j}, \quad T_{2}^{s}\left(\boldsymbol{a}^{s}\right)=\sum_{k=1}^{N_{m}} \sum_{l=1}^{N_{m}}\left(\int_{0}^{1} \phi_{k}^{\prime} \boldsymbol{\phi}_{l}^{\prime} \mathrm{d} x\right) \boldsymbol{a}_{k}^{s} \boldsymbol{a}_{l}^{s}, \\
& \boldsymbol{K}_{T i j}=\int_{0}^{1} \phi_{j}^{\prime \prime} \phi_{i} \mathrm{~d} x, \quad Q_{0 i}^{s}=\int_{0}^{1} \phi_{i} \mathrm{~d} x \\
& \boldsymbol{Q}_{1 i j}^{s}=-2 \int_{0}^{1} \phi_{i} \phi_{j} \mathrm{~d} x, \quad \boldsymbol{Q}_{2 i j}^{s}=3 \sum_{k=1}^{N_{m}}\left(\int_{0}^{1} \phi_{i} \phi_{j} \phi_{k} \mathrm{~d} x\right) \boldsymbol{b}_{k}^{s} \\
& \boldsymbol{Q}_{3 i j}^{s}=-4 \sum_{k=1}^{N_{m}} \sum_{l=1}^{N_{m}}\left(\int_{0}^{1} \phi_{i} \phi_{j} \phi_{k} \phi_{l} \mathrm{~d} x\right) \boldsymbol{b}_{k}^{s} \boldsymbol{b}_{l}^{s} \\
& \boldsymbol{Q}_{4 i j}^{s}=5 \sum_{k=1}^{N_{m}} \sum_{l=1}^{N_{m}} \sum_{m=1}^{N_{m}}\left(\int_{0}^{1} \phi_{i} \phi_{j} \phi_{k} \phi_{l} \phi_{m} \mathrm{~d} x\right) \boldsymbol{b}_{k}^{s} \boldsymbol{b}_{l}^{s} \boldsymbol{b}_{m}^{s} \\
& \boldsymbol{Q}_{5 i j}^{s}=-6 \sum_{k=1}^{N_{m}} \sum_{l=1}^{N_{m}} \sum_{m=1}^{N_{m}} \sum_{n=1}^{N_{m}}\left(\int_{0}^{1} \phi_{i} \phi_{j} \phi_{k} \phi_{l} \phi_{m} \phi_{n} \mathrm{~d} x\right) \boldsymbol{b}_{k}^{s} \boldsymbol{b}_{l}^{s} \boldsymbol{b}_{m}^{s} \boldsymbol{b}_{n}^{s} \\
& \boldsymbol{Q}_{6 i j}^{s}=7 \sum_{k=1}^{N_{m}} \sum_{l=1}^{N_{m}} \sum_{m=1}^{N_{m}} \sum_{n=1}^{N_{m}} \sum_{o=1}^{N_{m}}\left(\int_{0}^{1} \phi_{i} \phi_{j} \phi_{k} \phi_{l} \phi_{m} \phi_{n} \phi_{o} \mathrm{~d} x\right) \boldsymbol{b}_{k}^{s} \boldsymbol{b}_{l}^{s} \boldsymbol{b}_{m}^{s} \boldsymbol{b}_{n}^{s} \boldsymbol{b}_{o}^{s} \\
& \boldsymbol{P}_{3 i j}^{s}=-4 \sum_{k=1}^{N_{m}} \sum_{l=1}^{N_{m}}\left(\int_{0}^{1} \phi_{i} \phi_{j} \phi_{k} \phi_{l} \mathrm{~d} x\right) \boldsymbol{b}_{k}^{s-1} \boldsymbol{b}_{l}^{s-1} \\
& \boldsymbol{P}_{4 i j}^{s}=5 \sum_{k=1}^{N_{m}} \sum_{l=1}^{N_{m}} \sum_{m=1}^{N_{m}}\left(\int_{0}^{1} \phi_{i} \phi_{j} \phi_{k} \phi_{l} \phi_{m} \mathrm{~d} x\right) \boldsymbol{b}_{k}^{s-1} \boldsymbol{b}_{l}^{s-1} \boldsymbol{b}_{m}^{s-1}, \\
& \boldsymbol{P}_{5 i j}^{s}=-6 \sum_{k=1}^{N_{m}} \sum_{l=1}^{N_{m}} \sum_{m=1}^{N_{m}} \sum_{n=1}^{N_{m}}\left(\int_{0}^{1} \phi_{i} \phi_{j} \phi_{k} \phi_{l} \phi_{m} \phi_{n} \mathrm{~d} x\right) \boldsymbol{b}_{k}^{s-1} \boldsymbol{b}_{l}^{s-1} \boldsymbol{b}_{m}^{s-1} \boldsymbol{b}_{n}^{s-1}, \\
& \boldsymbol{P}_{6 i j}^{s}=7 \sum_{k=1}^{N_{m}} \sum_{l=1}^{N_{m}} \sum_{m=1}^{N_{m}} \sum_{n=1}^{N_{m}} \sum_{o=1}^{N_{m}}\left(\int_{0}^{1} \phi_{i} \phi_{j} \phi_{k} \phi_{l} \phi_{m} \phi_{n} \phi_{o} \mathrm{~d} x\right) \\
& \times \boldsymbol{b}_{k}^{s-1} \boldsymbol{b}_{l}^{s-1} \boldsymbol{b}_{m}^{s-1} \boldsymbol{b}_{n}^{s-1} \boldsymbol{b}_{o}^{s-1}, \\
& \boldsymbol{P}_{7 i j}^{s}=-8 \sum_{k=1}^{N_{m}} \sum_{l=1}^{N_{m}} \sum_{m=1}^{N_{m}} \sum_{n=1}^{N_{m}} \sum_{o=1}^{N_{m}} \sum_{p=1}^{N_{m}}\left(\int_{0}^{1} \phi_{i} \phi_{j} \phi_{k} \phi_{l} \phi_{m} \phi_{n} \phi_{o} \phi_{p} \mathrm{~d} x\right) \\
& \times \boldsymbol{b}_{k}^{s-1} \boldsymbol{b}_{l}^{s-1} \boldsymbol{b}_{m}^{s-1} \boldsymbol{b}_{n}^{s-1} \boldsymbol{b}_{o}^{s-1} \boldsymbol{b}_{p}^{s-1}, \\
& \text { with } i, j=1, . ., N_{m}
\end{aligned}
$$$$
\ddot{a}_{21}+c \dot{a}_{21}+\omega_{2} a_{21}+\beta_{21} a_{21}^{3}+\left(\beta_{22} \cos \Omega t+\beta_{23} \cos ^{2} \Omega t\right) a_{21}+
$$$$
\left(\delta_{21}+\delta_{22} \cos \Omega t+\delta_{23} \cos ^{2} \Omega t\right) a_{11}+
$$$$
\left(\gamma_{21}+\gamma_{22} \cos \Omega t+\gamma_{23} \cos ^{2} \Omega t\right)=0
$$

where

$$
\beta_{s 1}=151.35 \alpha_{1} ; \beta_{s 2}=-4 \alpha_{2}\left(V_{d c_{s, s+1}} V_{a c_{s, s+1}}+V_{d c_{s-1, s}} V_{a c_{s-1, s}}\right) ;
$$$$
\beta_{s 3}=-2 \alpha_{2}\left(V_{a c_{s-1, s}}^{2}+V_{a c_{s, s+1}}^{2}\right) ; \quad \delta_{11}=\delta_{21}=2 \alpha_{2} V_{d c_{12}}^{2} ;
$$$$
\delta_{12}=\delta_{22}=4 \alpha_{2} V_{d c_{12}} V_{a c_{12}}
$$$$
\delta_{13}=\delta_{23}=2 \alpha_{2} V_{a c_{12}}^{2} ; \gamma_{s 1}=0.83 \alpha_{2}\left(V_{d c_{s, s+1}}^{2}-V_{d c_{s-1, s}}^{2}\right) \text {; }
$$$$
\gamma_{s 2}=1.66 \alpha_{2}\left(V_{d c_{s, s+1}} V_{a c_{s, s+1}}-V_{d c_{s-1, s}} V_{a c_{s-1, s}}\right) \text {; }
$$$$
\gamma_{s 3}=0.83 \alpha_{2}\left(V_{a c_{s, s+1}}^{2}-V_{a c_{s-1, s}}^{2}\right)
$$

and $s=1,2$.

660 Let the relation between $\Omega$ and $\omega_{s}$ be

$\boldsymbol{Q}_{7 i j}^{s}=-8 \sum_{k=1}^{N_{m}} \sum_{l=1}^{N_{m}} \sum_{m=1}^{N_{m}} \sum_{n=1}^{N_{m}} \sum_{o=1}^{N_{m}} \sum_{p=1}^{N_{m}}\left(\int_{0}^{1} \phi_{i} \phi_{j} \phi_{k} \phi_{l} \phi_{m} \phi_{n} \phi_{o} \phi_{p} \mathrm{~d} x\right) \boldsymbol{b}_{k}^{s} \boldsymbol{b}_{l}^{s} \boldsymbol{b}_{m}^{s} \boldsymbol{b}_{n}^{s} \boldsymbol{b}_{o}^{s} \boldsymbol{b}_{p}^{s}$,

where $\omega_{s}$ is determined by

$$
\omega_{s}=\frac{\lambda_{s}^{4}-2 \alpha_{2}\left(V_{d c_{s, s+12}}^{2}+V_{d c_{s-1, s}}^{2}\right)}{1+\delta_{s_{0}}(s) m \phi_{1}\left(x_{0}\right)^{2}} .
$$

\section{Appendix C Three-beam array with asymmetric voltages}

The voltages used for the asymmetric three-beam array are given in Table 4 and the responses are plotted in Fig. 19.

Table 4 Asymmetric actuation voltages of the 3-beam array

\begin{tabular}{|c|c|c|c|c|}
\hline Design & $V d c_{10}(V)$ & $V d c_{21}(V)$ & $V d c_{32}(V)$ & $V d c_{n 3}(V)$ \\
& $V a c_{10}(V)$ & $V a c_{21}(V)$ & $V a c_{32}(V)$ & $V a c_{n 3}(V)$ \\
\hline$\# 5$ & 0.5 & 0.3 & 0.5 & 0.4 \\
& 0.5 & 0.3 & 0.5 & 0.4 \\
\hline
\end{tabular}

\section{Appendix B Averaging method for a two-beam array}

655 The Galerkin method with the fundamental mode is used as follows

$w_{1}(x, t)=\phi_{1}(x) a_{11}(t)$

$w_{2}(x, t)=\phi_{1}(x) a_{21}(t)$

and first-order Taylor series for the electrostatic forces as follows

$\frac{1}{\left(1+w_{s+1}-w_{s}\right)^{2}}=1-2\left(w_{s+1}-w_{s}\right)$
$\frac{1}{\left(1+w_{s}-w_{s-1}\right)^{2}}=1-2\left(w_{s}-w_{s-1}\right)$.

Eq. (15) becomes

$\ddot{a}_{11}+c \dot{a}_{11}+\omega_{1} a_{11}+\beta_{11} a_{11}^{3}+\left(\beta_{12} \cos \Omega t+\beta_{13} \cos ^{2} \Omega t\right) a_{11}+$

$\left(\delta_{11}+\delta_{12} \cos \Omega t+\delta_{13} \cos ^{2} \Omega t\right) a_{21}+$

$$
\left(\gamma_{11}+\gamma_{12} \cos \Omega t+\gamma_{13} \cos ^{2} \Omega t\right)=0
$$



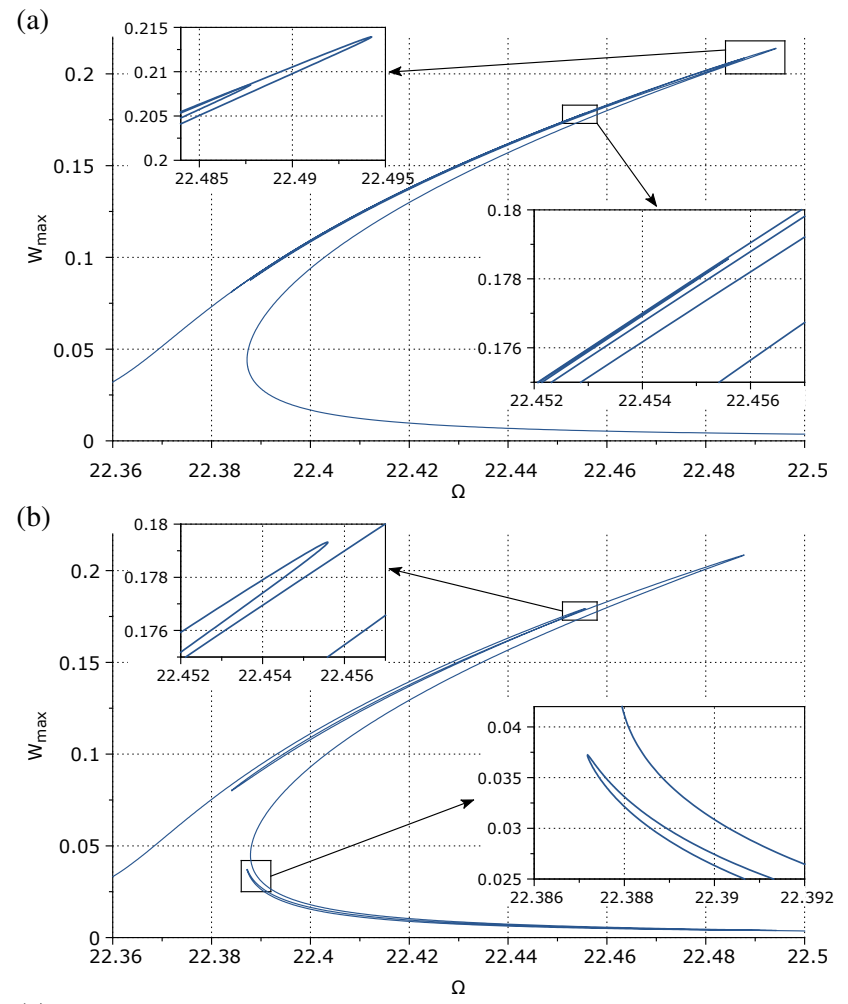

(c)

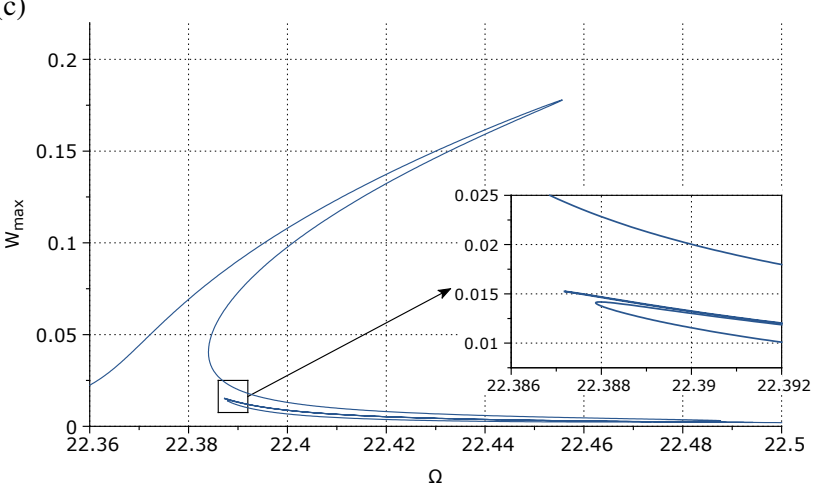

Fig. 19 Design \# 4. Responses of the three-beam array without added mass. (a): Beam \#1; (b): Beam \#2; (c): Beam \#3. 